This is a self-archived version of an original article. This version may differ from the original in pagination and typographic details.

Author(s): Luomala, Jari; Hakala, Ismo

Title: Analysis and Evaluation of Adaptive RSSI-based Ranging in Outdoor Wireless Sensor Networks

Year: 2019

Version: Accepted version (Final draft)

Copyright: (c) 2018 Elsevier B.V.

Rights: CC BY-NC-ND 4.0

Rights url: https://creativecommons.org/licenses/by-nc-nd/4.0/

Please cite the original version:

Luomala, J., \& Hakala, I. (2019). Analysis and Evaluation of Adaptive RSSI-based Ranging in Outdoor Wireless Sensor Networks. Ad Hoc Networks, 87, 100-112.

https://doi.org/10.1016/j.adhoc.2018.10.004 


\section{Accepted Manuscript}

Analysis and Evaluation of Adaptive RSSI-based Ranging in Outdoor Wireless Sensor Networks

Jari Luomala, Ismo Hakala

PII: S1570-8705(18)30729-7

DOI: https://doi.org/10.1016/j.adhoc.2018.10.004

Reference: ADHOC 1778

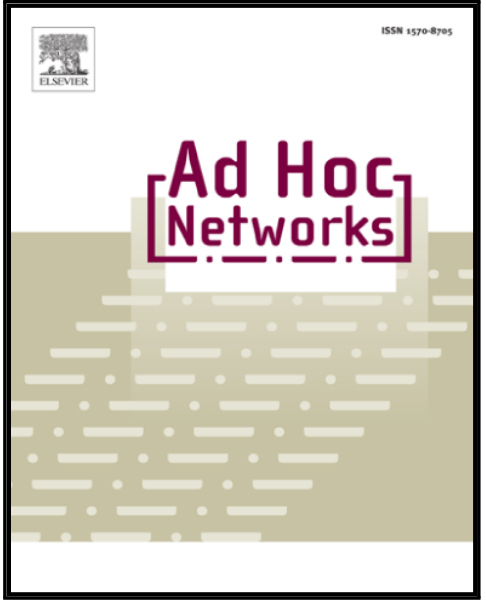

To appear in:

Ad Hoc Networks

Received date:

12 March 2018

Revised date:

6 September 2018

Accepted date:

10 October 2018

Please cite this article as: Jari Luomala, Ismo Hakala, Analysis and Evaluation of Adaptive RSSI-based Ranging in Outdoor Wireless Sensor Networks, Ad Hoc Networks (2018), doi: https://doi.org/10.1016/j.adhoc.2018.10.004

This is a PDF file of an unedited manuscript that has been accepted for publication. As a service to our customers we are providing this early version of the manuscript. The manuscript will undergo copyediting, typesetting, and review of the resulting proof before it is published in its final form. Please note that during the production process errors may be discovered which could affect the content, and all legal disclaimers that apply to the journal pertain. 


\title{
Analysis and Evaluation of Adaptive RSSI-based Ranging in Outdoor Wireless Sensor Networks
}

\author{
Jari Luomala*, Ismo Hakala \\ University of Jyvaskyla, Kokkola University Consortium Chydenius \\ P.O. Box 567, FI-67701 Kokkola, Finland
}

\begin{abstract}
Estimating inter-node distances based on received radio signal strength (RSSI) is the foundation of RSSI-based outdoor localization in wireless sensor networks (WSNs). However, the accuracy of RSSI-based ranging depends on environmental and weather conditions. Therefore, it is important that RSSI-based ranging adapts to prevailing conditions to improve its range and location accuracy. This paper analyzes and evaluates RSSI-based ranging and adaptive techniques in outdoor WSNs to improve the range quality. The findings highlight the effects of path loss exponent (PLE) estimation error and temperature change on RSSI-based ranging. Consequently, we analyze techniques for mitigating these detrimental effects and propose an adaptive RSSI-based ranging algorithm in order to improve the ranging quality in changing outdoor conditions. The algorithm comprises link RSSI estimation, temperature compensation, PLE estimation, and inter-node distance estimation. Furthermore, we evaluate the performance of the proposed algorithm and compare different WSN-specific PLE estimation techniques by employing real measurement data of 2.4 GHz IEEE 802.15.4-compliant WSN nodes. The results indicate that although ranging error can be mitigated using the proposed adaptive techniques, the accuracy when a single PLE estimate is used is, in general, limited due to high inter-link PLE variation.
\end{abstract}

Keywords: RSSI-based ranging, adaptive, localization, wireless sensor network, path loss exponent, temperature

\section{Introduction}

Location information about sensor nodes is an integral part of most sensor data in making it meaningful and is needed for many functions, applications, and services in wireless sensor networks (WSNs), such as target tracking, geographic routing, and location-based services [1-6]. Typically, only a few WSN nodes know their locations a priori (e.g., by using the Global Navigation Satellite System, GNSS) and are called reference nodes (aka anchors, beacons, and landmarks). Nodes with unknown locations usually try to locate themselves by using distance estimates to reference nodes and the reference nodes' coordinates. This kind of anchor- and range-based localization is commonly used in WSNs.

The foundation of any range-based localization algorithm is the estimation of physical distances between two sensor nodes based on characteristics of the signals exchanged between the nodes [1]. Ranging is a key factor in localization, and the location accuracy is largely determined by the quality of the ranging $[5,7]$. Different ranging techniques (Received Signal Strength Indicator (RSSI), Time of Arrival (ToA), Time Difference of Arrival (TDoA), and Angle of Arrival (AoA)) are generally employed to estimate inter-node distances [1, 2, 4, 5]. RSSI-based ranging, based on radio signal strength obtained with the help

\footnotetext{
*Corresponding author

Email addresses: jari.luomala@chydenius.fi (Jari Luomala), ismo.hakala@chydenius.fi (Ismo Hakala)
}

of a received signal strength indicator, is a tempting, low-cost choice and is relatively easy to implement because no extra hardware is needed. However, the method has its drawbacks; RSSI-based ranging is highly sensitive to changes in environmental and weather conditions, which may result in reduced location accuracy.

As is well-known, the attenuation and behavior of radio signals are highly environment-specific. Therefore, using a path loss model that does not reflect the signal attenuation correctly in a particular propagation environment may lead to considerable ranging errors. Specifically, the path loss exponent (PLE) is the single most important parameter affecting ranging accuracy. Furthermore, changing weather conditions may cause much variation in the radio signal strength of wireless sensor nodes. Temperature variation in particular is one of the major individual factors affecting radio signal strength in outdoor WSNs [8-13]. Consequently, the performance of several algorithms, protocols, and services that utilize signal strength will suffer if the effects of ambient temperature are ignored. RSSIbased ranging and localization are at the top of this list. In addition to these causes, other factors affect RSSI-based ranging. These include, for example, RF transceiver non-idealities, antenna characteristics, and various interference sources.

These challenges require the use of robust, adaptive techniques in order to achieve sufficient location accuracy and precision regardless of the prevailing conditions. Typically, a WSN consists of low-cost, resource-constrained nodes with 
limited communication, energy, memory, and processing capacity. Therefore, the complexity of the proposed methods should be relatively low, in terms of computation and communication. Computation should be able to be performed mostly in a distributed fashion in the nodes, as sending data to the sink/server for computation consumes resources and is errorprone.

This paper aims to find means of improving the RSSI-based ranging quality of WSN nodes in varying outdoor conditions. Based on empirical and analytical results, we show that path loss exponent estimation error and temperature change may have marked detrimental effects on the ranging quality. As our first attempt to mitigate these effects and to improve ranging quality, we analyze link RSSI estimation, temperature compensation, and PLE estimation techniques for adaptive RSSI-based ranging. The PLE estimation techniques selected for comparison (averaging, least-squares estimation, optimization) are basic, low-complex techniques for this size problem, and can be implemented in resource-constrained nodes. As a result, we propose an adaptive RSSI-based ranging algorithm that integrates these techniques with inter-node distance estimation aiming to mitigate RSSI/PLE variation and PLE estimation error and, consequently, ranging error.

In the algorithm, reference nodes compute PLEs for reference links based on temperature-compensated RSSI data once at the beginning (e.g., based on 24 hours data), and then interchange the PLEs and other relevant data. Next, reference nodes estimate a WSN-specific PLE to be sent to nodes. To estimate the distance to some other node, a node applies the WSNspecific PLE and temperature-compensated RSSI data for the link. We evaluate the performance of the adaptive RSSI-based ranging algorithm and compare different WSN-specific PLE estimation techniques by employing real measurement data of $2.4 \mathrm{GHz}$ stationary WSN nodes. Overall, the results provide useful information for RSSI-based localization in WSNs, particularly when it is employed in varying outdoor conditions.

In summary, this study makes the following main contributions:

- We show the effects of path loss exponent estimation error and temperature change on RSSI-based ranging.

- We analyze link RSSI estimation, temperature compensation, and PLE estimation techniques for adaptive RSSIbased ranging.

- We propose an adaptive RSSI-based ranging algorithm to improve the ranging quality.

- We evaluate the performance of the adaptive RSSI-based ranging algorithm and compare different PLE estimation techniques by employing real measurement data.

The rest of this paper is organized as follows. In Section 2, we briefly highlight several related studies. In Section 3, we present the application of an RSSI-based ranging technique, and Section 4 follows with a short description of the experimental measurements. Based on the results, in Section 5, we analyze the main sources of error in RSSI-based ranging. In the pursuit of better ranging quality, we propose an adaptive RSSIbased ranging algorithm in Section 6 . In Section 7, we evaluate the performance of the adaptive RSSI-based ranging algorithm using different PLE estimation techniques, followed by a short discussion in Section 8. Finally, we conclude our work in Section 9.

\section{Related Work}

The challenges of RSSI-based localization have motivated researchers to find robust, adaptive techniques for ranging that consider different environments and their dynamics. Many types of methods for (adaptive) RSSI-based ranging have been studied in the literature recently. Most of these methods try to estimate the parameters (usually the PLE) of the ranging model for a particular environment. In the following, we look at some research related to ours.

In [14], the authors investigated different factors affecting RSSI-based ranging quality in WSNs and evaluated the use of statistical methods and artificial neural networks for RSSIbased ranging. Some adaptive RSSI-based ranging methods that use the log-normal shadowing model (LNSM), such as [15] and [16], estimate or calibrate the coefficients in the model with a technique in order to dynamically adapt to different and changing environments. In [7], the authors instead proposed a combined and differentiated localization (CDL) approach that integrates local filtration techniques and ranging-quality aware calibration to improve the ranging quality.

Much of the literature on RSSI-based ranging emphasizes the importance of the path loss exponent for ranging quality. A conventional technique is to assume that the PLE is known a priori for the particular environment, or the PLE is computed offline based on an extensive set of RSSI and distance measurements before the PLE is deployed in the WSN [17]. Recently, more sophisticated methods for estimating the PLE online have been proposed. Some PLE estimation methods, such as [17-20], do not require distance measurements or other nodes' locations. PLE estimation of those methods can be based only on RSS measurements if the probability distribution of the inter-node distances is known [17], geometric constraints of the WSN [17], mean interference in the network [18], virtual outage probabilities [18], neighborhood size [18], or RSS distribution [20]. Although these methods do not need distance measurements in PLE estimation, depending on the method, they make assumptions, such as previous knowledge of the distance distribution, network density, node distribution, channel model/fading, RSS distribution, or other network parameters. These assumptions may be unrealistic and thus, non-generic in many real scenarios. The disadvantage of some methods, such the one proposed in [17], is high computational complexity making the method unsuitable for distributed implementation. Most of the methods also assume an omnidirectional PLE while a few estimate a directional PLE [20]. Contrary to these techniques, many of the existing PLE estimation methods apply distance measurements and reference nodes. Some methods estimate the PLE in the localization phase iteratively and alternately with node locations $[21,22]$. They can use, e.g., the PLE from reference nodes as 
an initial value to compute the node location [21], or even an unknown PLE [22], which is then used to recompute the PLE. In [23], the authors proposed a distributed, cooperative method that minimizes the cost function of unknown nodes' coordinates and PLEs using the iterative gradient descent approach. The study in [24] also applied the gradient descent technique, but the authors mainly emphasized the importance of measurement arrangements for accurately estimating the PLE. Instead of using a fixed PLE value, it is possible to define a value scope of PLEs that is then used to create an RSSI-based ranging scope to be used in localization [25].

Compared to the PLE, the body of literature on the effect of temperature on RSSI-based ranging and localization is small. In recent years, however, the effects of temperature on the radio signal strength of WSN nodes have been explored and recognized in several studies, such as [8-13]. Clearly, the temperature affects RSSI-based ranging and localization outdoors. Bannister et al. [8] studied how temperature affects signal strength and different services, including RSSI-based ranging and localization, in WSNs. The authors showed that temperature has a negative linear effect on the RSS which may lead even to a $150 \%$ ranging error when the RSS is applied to localization without temperature compensation. They suggested including temperature sensors in nodes to compensate the RSS variation caused by temperature and to reduce ranging and localization errors. However, we recognize that temperature compensation is not necessary for ranging if the PLE estimation is computed frequently. However, PLE estimation is not cost-effective as it increases computation and communication. Moreover, temperature compensation of RSSI values may be useful for purposes other than ranging.

Another factor affecting RSSI-based ranging is the channelspecific variation in RSSI values (due to multipath, noise, interference, etc.). A potential mitigation method for high RSSI variation is frequency diversity given that multiple channels are employed. A multichannel approach for improving RSSI-based ranging accuracy has been studied, for example, in [26] and [27].

Most studies in the field of RSSI-based ranging have mainly focused on the effect of the PLE and ignored the effect of temperature or other weather variables which may change rapidly. Furthermore, many of the existing approaches make strong assumptions about some network parameters that need to be known a priori, or the approaches are computationally too complex requiring centralized implementation. Most are also verified based on simulations only. The adaptive RSSI-based ranging algorithm we propose combines frequency diversity and temperature compensation with online PLE estimation in aiming to improve ranging quality in fluctuating outdoor conditions. The algorithm is robust, adapting its operation based on the ambient conditions and temperature, and is designed and verified based on real measurement data. The proposed algorithm is low cost in terms of computation and communication, and can be implemented in typical resource-constrained sensor nodes in a distributed fashion.

\section{RSSI-based Ranging Model}

Inter-node distances needed in range-based localization can be computed based on signal strength measurements by using an RSSI-based ranging technique. The central idea of exploiting radio signal strength in ranging is that signal strength decays as a function of the distance between the transmitter and the receiver $\left(R S S \propto d^{-n}\right)$ [1]. Several generally used path loss models that can be applied to RSSI-based ranging differ in accuracy, methods, and goals, for example.

A well-known model generally used to reflect the path loss of a radio signal is the log-normal shadowing model (see, e.g., [28]). The model is used as the basis of this study. It can be expressed in $\mathrm{dBm}$ as:

$$
\begin{gathered}
P_{r}(d)=\bar{P}_{r}(d)+X_{\sigma}, \\
\bar{P}_{r}(d)=P_{r}\left(d_{0}\right)-10 n \log \left(\frac{d}{d_{0}}\right),
\end{gathered}
$$

where $P_{r}(d)$ is the received power $[\mathrm{dBm}]$ at distance $d[\mathrm{~m}]$ from the transmitter, $\bar{P}_{r}(d)$ is the corresponding average power $[\mathrm{dBm}], X_{\sigma}$ is the zero-mean Gaussian random variable with the variance of $\sigma^{2}\left(N\left(0, \sigma_{\mathrm{dB}}\right)\right), P_{r}\left(d_{0}\right)$ is the received power $[\mathrm{dBm}]$ at the reference distance $d_{0}$ (usually $1 \mathrm{~m}$ ) from the transmitter, and $n$ is the path loss exponent. $P_{r}\left(d_{0}\right)$ can be a predefined or measured value, or it can be estimated by using the Friis freespace equation as:

$$
P_{r}\left(d_{0}\right)=\frac{P_{t} G_{t} G_{r} \lambda^{2}}{(4 \pi)^{2} d_{0}^{2} L},
$$

where $P_{t}$ is the transmission power [mW], $G_{t}$ and $G_{r}$ are the antenna gains $[\mathrm{mW}]$ of the transmitting and receiving antennas, respectively, $\lambda$ is the wavelength, and $L \geq 1$ is the sum of losses through the transmit/receive circuitry. The PLE $n$ depends on the specific propagation environment (usually between 2 and 4 [4]) and can be predefined, or computed offline or online by using fixed nodes with known distances, in some cases even without distance information (see, e.g., [17-20]).

Model (2) without the stochastic term is often referred to as the log-distance path loss model. It can be used for RSSI-based ranging to estimate the distance, $\hat{d}[\mathrm{~m}]$ (maximum likelihood estimate, MLE), between two neighboring nodes as follows:

$$
\hat{d}=d_{0} 10^{\left(P_{r}\left(d_{0}\right)-\bar{P}_{r}(d)\right) / 10 n} .
$$

As can be seen, the accuracy of distance estimate $\hat{d}$ depends on the accuracy of the signal power and the PLE used. If they are accurate, then the distance estimate is accurate. However, in practice, some error is included in them, thus resulting in a varying ranging error.

Ranging error $e_{d}$, relative to the true distance, can be denoted as:

$$
e_{d}=\frac{\Delta d}{d}=\frac{\hat{d}-d}{d},
$$

where $\hat{d}$ is the distance estimate, and $d$ is the true distance [m]. 


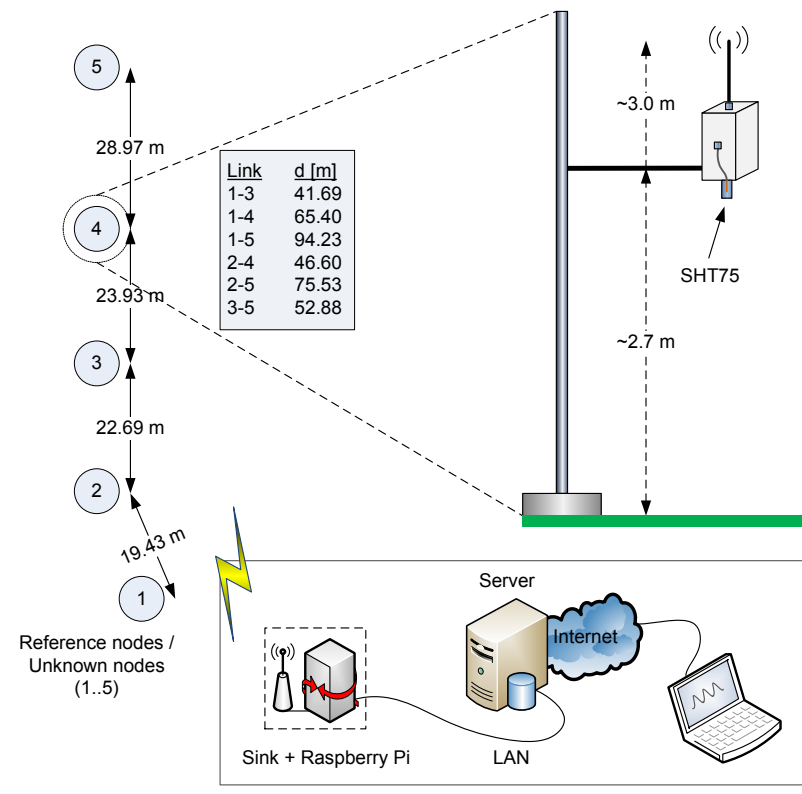

Figure 1: Experimental setup (not to scale).

\section{Experimental Measurement}

The WSN that collected the empirical measurement data for the analysis and evaluation consisted of Atmel ZigBit $2.4 \mathrm{GHz}$ wireless modules [29] with an IEEE 802.15.4compliant AT86RF230 radio transceiver [30]. Each node was integrated with a Sensirion humidity and temperature sensor (SHT75) [31] to measure the ambient temperature and relative humidity. The test equipment consisted, in total, of five sensor nodes, one sink node, one Raspberry $\mathrm{Pi}$, and one server/database. The sensor nodes were attached to lamp posts around the university campus, next to the parking lot, while the other equipment was inside the university building. There was a direct line-of-sight (LOS) propagation path between the nodes without obstacles. However, some objects were in close proximity (e.g., trees, cars, a traffic sign) which may have altered the radio signal, for example, due to reflection, diffraction, and scattering. The network setup is illustrated in Figure 1.

RSSI data for the links was collected, once a minute, with two different transmit power levels, $+3.0 \mathrm{dBm}\left(\mathrm{P}_{\mathrm{TX} 1}\right)$ and $-7.2 \mathrm{dBm}\left(\mathrm{P}_{\mathrm{TX} 2}\right)$. The radio channel (11-26) was changed every minute. In addition, the temperature readings for each node were measured once a minute with the SHT75 sensors. The collected raw data was sent to the sink, from where the data was forwarded via Raspberry Pi to the server/database to be further processed and analyzed with MATLAB. The RSSI data of each channel was averaged over $1 \mathrm{~h}$. These $1 \mathrm{~h}$ RSSI samples collected at different radio channels were used to compute the link RSSI by using the methods described in Section 6. In addition, the temperature data was averaged over $1 \mathrm{~h}$. Two-way link RSSI was computed by averaging the link RSSI values of both nodes. These two-way link RSSI $1 \mathrm{~h}$ average values were used in the analysis and evaluation.

The experimental measurement data used in the analysis and evaluation was collected during 1 week between July 21 and 27 ,
2014. Due to the summer vacation period, traffic in the parking lot was sparse at that time. The total number of samples of the RSSI and temperature data was 194543 (96.5\% Packet Reception Ratio, PRR) and 48612 (96.5\% PRR), respectively. This denotes 38909 RSSI samples and 9722 temperature samples per node, on average. The number of $1 \mathrm{~h}$ RSSI samples was 53673, which denotes 10735 samples per node, 2684 samples per link, and 168 samples per each link channel, on average. The number of $1 \mathrm{~h}$ temperature samples was 840 , which denotes 168 samples per node, on average.

\section{Sources of Error in RSSI-based Ranging}

The main challenge with RSSI-based ranging is the sensitivity of the radio signal strength to changes in environmental conditions which arises as a variation in RSSI values. This variation may be caused by external factors, such as the propagation environment, weather conditions, interference, etc. In addition, variations in RSSI can be partly due to internal factors of the node hardware. Variations in RSSI values further result in variations in the path loss exponent within and between the links, and thus, errors in estimating the path loss exponent for ranging purposes.

In this section, we analyze two of the main sources of errors in RSSI-based ranging, both theoretically and based on the empirical results when the ranging model is employed. These sources are (i) an error in the path loss exponent estimation and (ii) temperature change. Furthermore, several other sources of error are identified.

\subsection{Path Loss Exponent Estimation Error}

The path loss exponent, which measures the rate at which the signal strength decays with distance, is a key factor in RSSIbased ranging and localization and depends on the particular propagation environment [17]. Errors in the PLE estimation may have a big impact on ranging errors. Figure 2 (a) illustrates theoretically how the PLE estimation error (difference from $n_{\text {true }}$ ) affects the ranging error when the log-distance path loss model in (4) is used. As can be seen, the ranging error (5) increases quite a bit if the estimated PLE does not reflect the true path loss. The magnitude of the ranging error depends on which side of the true PLE $(n)$ the estimated PLE $(\hat{n})$ resides. As shown in Figure 2 (a), a theoretical overestimation of the PLE results in a considerably lower ranging error than underestimation by an equal amount. For example, if it is assumed that $n=2.2$, using $\hat{n}=2.4$ in ranging results in a $-26.5 \%$ $(-10.6 \mathrm{~m})$ ranging error for the $40 \mathrm{~m}$ link, while using $\hat{n}=2.0$ results in a $44.6 \%(17.8 \mathrm{~m})$ ranging error, respectively. A similar effect of PLE estimation error was observed in [17] for a WSN position estimation error. Furthermore, the PLE error has a smaller impact on the ranging error when the inter-node distances are smaller. For example, in Figure 2 (a), $\hat{n}=2.0$ results in a $17.5 \%(0.9 \mathrm{~m})$ ranging error for the $5 \mathrm{~m}$ link but a $55.0 \%(44.0 \mathrm{~m})$ ranging error for the $80 \mathrm{~m}$ link. Therefore, the distances between the links used in ranging may have a huge impact on ranging errors. 


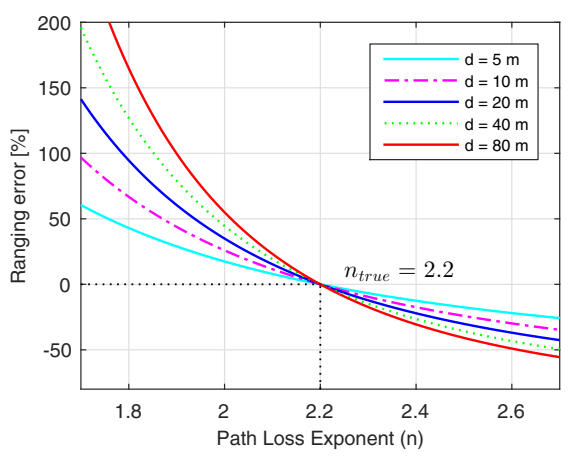

(a)

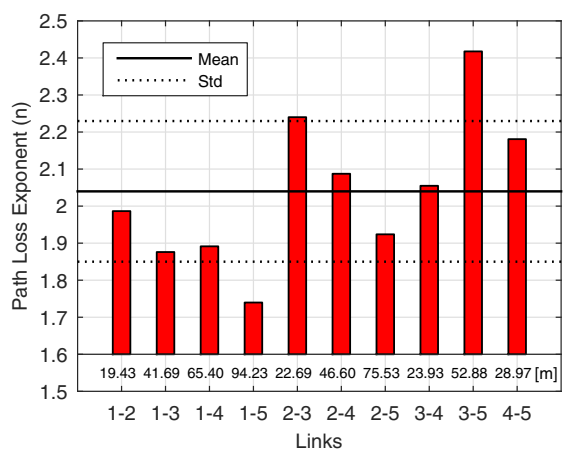

(b)

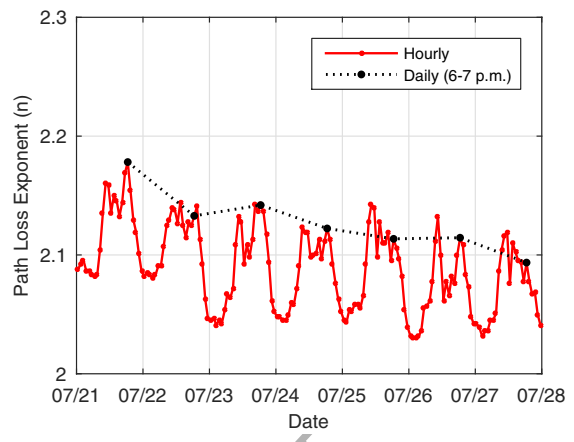

Figure 2: (a) The effect of the PLE estimation error on the ranging error when the log-distance path loss model is used at distances $d=\{5,10,20,40,80\}$ m. $P_{r}(d)$ was computed using $n=2.2$ at the particular distance, and in estimating $\hat{d}$, it was kept fixed while $n$ was changed. (b) The PLEs of the one-way links computed at the same $1 \mathrm{~h}$ time period (in July 21,2014 ) at night time ( $1 \mathrm{~h}$ RSSI values averaged over 16 channels), (c) the PLE of the link $2 \rightarrow 4$ during $1 \mathrm{week}$ in July $21-27$, 2014 (hourly and daily). In each case, transmit power $\mathrm{P}_{\mathrm{TX}}=-7.2 \mathrm{dBm}$.

\begin{tabular}{c|c|c||c|c|c}
\hline Link & $\Delta R S S I[\mathrm{~dB}]$ & $\Delta P L E$ & Link & $\Delta R S S I[\mathrm{~dB}]$ & $\Delta P L E$ \\
\hline \hline $1-2$ & 0.36 & 0.03 & $2-4$ & 1.26 & 0.08 \\
$1-3$ & 0.50 & 0.03 & $2-5$ & 0.81 & 0.04 \\
$1-4$ & 0.39 & 0.02 & $3-4$ & 1.41 & 0.10 \\
$1-5$ & 0.09 & 0.00 & $3-5$ & 0.35 & 0.02 \\
$2-3$ & 0.47 & 0.03 & $4-5$ & 0.87 & 0.06 \\
\hline
\end{tabular}

Table 1: Differences in RSSI/PLE between opposite directions based on the RSSI values averaged over the channels for the same $1 \mathrm{~h}$ time period (in July 21, 2014).

The propagation environment and the inter-node distance affect the path loss exponent. Even inside a WSN, in a relatively small area with line-of-sight conditions and links pointing nearly at the same angle, PLEs between different links may vary quite a lot, as can be seen in Figure 2 (b). For the particular links and time, using the average PLE would lead to ranging errors between $-48.8 \%(-45.95 \mathrm{~m})$ and $108.6 \%(57.40 \mathrm{~m})$ for the links $1 \rightarrow 5$ and $3 \rightarrow 5$, respectively. Therefore, using the same PLE for each link is challenging and may cause large ranging errors unless the variation in the PLE between the links is mitigated. Additionally, the PLEs of two-way links also have different variations between the opposite directions. In Table 1, the differences ( $\triangle R S S I, \triangle P L E)$ between the opposite directions of the links at the same time as in Figure 2 (b) are shown (1 h averaged over the channels). As can be seen, the differences vary quite a lot depending on the link. $\triangle P L E$ varies from 0.00 to 0.10 , which is considerable in terms of the ranging error. The differences likely derive mostly from hardware-related issues, such as different transmit powers.

Furthermore, the PLE of a single link may have short- and long-term variations, illustrated in Figure 2 (c). For this link, a short-term (daily) variation in the PLE appears to be larger than long-term (weekly) variations. Assuming that the nodes are stationary, as in our case, a variation in the PLE is the direct result of a variation in the RSSI (path loss). To sum up, the PLE features spatial inter-link variations and temporal intralink variations, which result from variations in the RSSI/path loss. Overall, these results indicate that mitigating the PLE es- timation error is the main task in the pursuit of better ranging accuracy.

\subsection{Temperature Change}

Previous studies $[8,12,13]$, including ours, have shown that temperature has a negative, approximately linear effect on radio signal strength (RSSI). An increase in the temperature causes the RSSI to fall, and vice versa, as illustrated in Figure 3 (a). This means that the RSSI-based ranging accuracy also changes with the temperature change if not compensated. Figures 3 (b) and (c) illustrate theoretically how the temperature change affects the RSSI-ranging error when the log-distance path loss model in (4) is used with the empirically estimated temperature effect. In Figure 3 (b), if it is assumed that the measured inter-node distance of the $50 \mathrm{~m}$ link is correct when the temperature is $25^{\circ} \mathrm{C}$, a $15^{\circ} \mathrm{C}$ decrease and increase in temperature cause ranging errors of $-8.1 \mathrm{~m}$ and $+9.7 \mathrm{~m}$, respectively. As can be seen in Figure 3 (c), a change in temperature has a considerable effect on the ranging error (tens of percent), and it is emphasized with lower path loss exponents and higher temperatures.

There is also an apparent relation between the temperature and the PLE. A temperature change causes an RSSI change, which results in a PLE change. Therefore, the short-term intralink variation in the PLE can be mostly explained by the temperature change. At a specific time, the effect of the temperature is included in the computed path loss exponent, but it does not apply if the temperature changes. Consequently, either temperature compensation or PLE recomputation is necessary.

\subsection{Other Sources of Error}

The effects of the PLE estimation error and the temperature change on the ranging error can be partly mitigated, as will be shown. However, other factors remain that produce ranging errors and that need to be mitigated by other means. These other sources of error include RF transceiver non-idealities, antenna characteristics, and various interference sources. In addition, other weather conditions, such as humidity and rain/snow, in addition to temperature, may have an effect [13]. Reduction in 

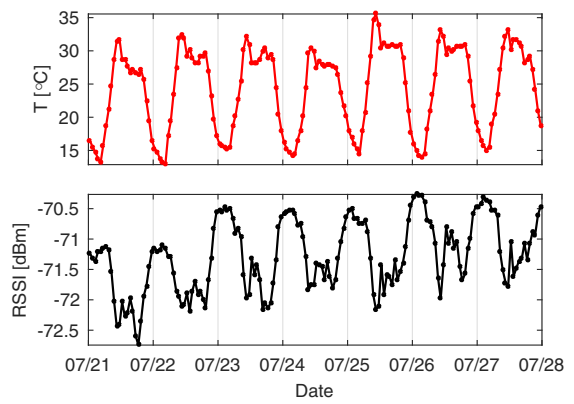

(a)

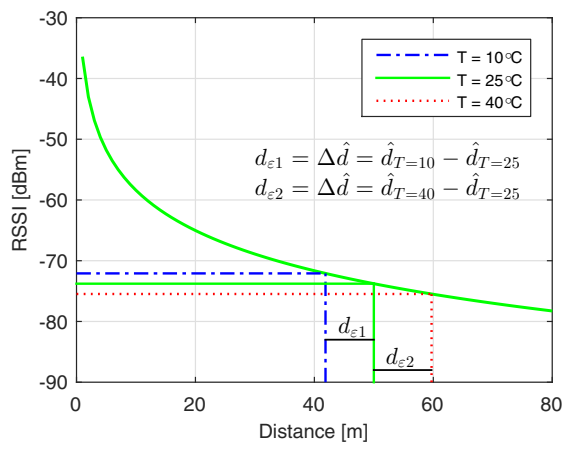

(b)

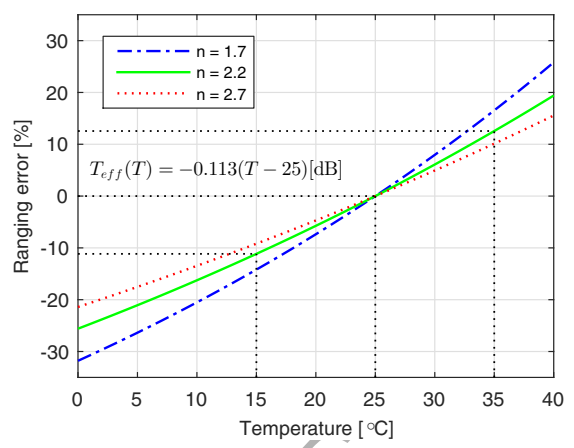

(c)

Figure 3: (a) The variation in the temperature and the RSSI (averaged over the channels) for a link (2 $\rightarrow$ 4) during 1 week (July $21-27$, 2014), (b) RSSI vs. distance when the $\log$-distance path loss model $(n=2.2)$ is used at temperatures $T=\{10,25,40\}^{\circ} \mathrm{C}$, (c) the effect of the temperature on the ranging error when the log-distance path loss model is used with path loss exponents $n=\{1.7,2.2,2.7\}$. In each case, transmit power $\mathrm{P}_{\mathrm{TX}}=-7.2 \mathrm{dBm}$.

the stochastic fluctuation of the RSSI can be implemented by averaging, as we do, or filtering out with an appropriate technique. A channel-specific variation (due to multipath, noise, interference, etc.) could be partly mitigated by using frequency diversity [13, 32] if multiple channels are employed. In [26], it was shown that by averaging RSS samples collected on different radio channels it is possible to increase RSS-based ranging accuracy. An example of channel-specific RSSI variation of link $2 \rightarrow 4$ for 1 week is shown in Table 2 . The inter-link PLE variation, shown in Table 3, is also dependent on the channel used, but we discuss this topic further in the next section.

\section{Adaptive RSSI-based Ranging}

As shown in the previous section, temperature change and path loss exponent estimation error in particular may cause a severe deterioration in the ranging accuracy if not compensated somehow. In the pursuit of better ranging quality, we survey a set of methods and techniques to adapt to changing environmental and weather conditions in order to mitigate these detrimental effects. The proposed methods can then be applied in RSSI-based ranging with the log-distance path loss model, and their effect on the ranging accuracy can be evaluated.

The proposed adaptive RSSI-based ranging algorithm, depicted as a flow chart in Figure 4, comprises (i) link RSSI estimation, (ii) temperature compensation, (iii) PLE estimation, and (iv) inter-node distance estimation. The first phase of the algorithm aims to mitigate the spatial inter-link PLE variation by selecting the most appropriate radio channels (as for ranging) for each link. It also mitigates the node-specific RSSI variation of each two-way link. In the second phase, temperature compensation is applied to RSSI values to mitigate the temporal intra-link RSSI/PLE variation caused by temperature variation. In the third phase, we try to estimate a WSNspecific PLE based on reference links' PLEs, computed based on temperature-compensated RSSI data during some period ( 24 hours in our case). The aim is to mitigate the error between the WSN-specific PLE applied in ranging and the true PLE for each link. Finally, in the fourth phase, inter-node distance is estimated by applying the log-distance path loss model with link
RSSI estimation, temperature compensation, and PLE estimation. The proposed algorithm considers spatial and temporal aspects of RSSI variation. The solution is modular and feasible to implement in resource-constrained WSN nodes. In the following subsections, we describe and analyze the different phases of the algorithm in detail. Furthermore, we briefly discuss implementing the algorithm in WSN nodes.

\subsection{Link RSSI Estimation}

The aim of link RSSI estimation is to mitigate the spatial inter-link PLE variation by selecting the most appropriate radio channels relative to the ranging for each link. By narrowing the range of PLEs between links, we can reduce PLE estimation error and therefore, the ranging error. If the same fixed channel is used in ranging for all the links, the difference in the PLEs between links would vary heavily depending on the channel, as can be seen in Table 3 where the links' mean PLEs based on 1 week using fixed channels are compared. The standard deviation $\sigma$ of the links' mean PLEs $(n=20)$ varies between 0.14 (ch13) and 0.32 (ch19), and the range $\Delta\left(P L E_{\max }-P L E_{\min }\right)$ from 0.50 even to as high as 1.05 . This would inevitably lead to large ranging errors.

To mitigate the channel-specific variation of each link (due to multipath, noise, interference, etc.), we applied several alternative techniques instead of a single arbitrary channel. The techniques were (E1) the average of 16 channels RSSI, (E2) the best RSSI ( $\max R S S I$ ), and (E3) the weighted mean of the best 3 RSSI ( $\max _{3} R S S I$ ) values, for each link based on $1 \mathrm{~h}$ values, expressed formally as:

$$
\begin{aligned}
& R S S I_{1 \mathrm{~h}}=\frac{1}{16} \sum_{i=11}^{26} \overline{\operatorname{RSSI}}_{i}, \\
& R S S I_{1 \mathrm{~h}}=\max \overline{\operatorname{RSSI}}_{i}, \\
& \operatorname{RSSI}_{1 \mathrm{~h}}=\frac{1}{\sum_{j=1}^{3} w_{j}} \sum_{j=1}^{3} w_{j} \max _{3} \overline{\operatorname{RSSI}}_{i},
\end{aligned}
$$

where $i=11 \ldots 26$ is the radio channel, $\overline{R S S I}_{i}$ is its $1 \mathrm{~h}$ average, and $w_{j}$ are the weights for the $\max _{3} \overline{R S S I}_{i}$ values in 


\begin{tabular}{l|r|r|r|r|r|r|r|r|r|r|r|r|r|r|r|r}
\hline \multicolumn{1}{c|}{ Ch } & 11 & 12 & 13 & 14 & 15 & 16 & 17 & 18 & 19 & 20 & 21 & 22 & 23 & 24 & 25 & 26 \\
RSSI [dBm] & & & & & & & & & & & & & & & \\
\hline \hline$\mu$ & -71.75 & -70.71 & -70.02 & -70.02 & -74.24 & -67.84 & -71.40 & -72.63 & -71.32 & -70.28 & -75.46 & -72.05 & -68.26 & -71.98 & -73.47 & -68.96 \\
$\sigma[\mathrm{dB}]$ & 0.75 & 0.70 & 0.47 & 0.51 & 0.90 & 0.75 & 0.82 & 1.01 & 0.57 & 0.66 & 1.07 & 0.84 & 0.85 & 1.09 & 0.74 & 0.83 \\
\hline $\max$ & -70.25 & -69.00 & -68.67 & -68.75 & -72.50 & -66.67 & -70.00 & -71.00 & -70.00 & -68.75 & -74.00 & -70.00 & -66.75 & -69.75 & -72.00 & -67.00 \\
$\min$ & -74.00 & -73.00 & -72.25 & -71.67 & -76.33 & -69.50 & -73.33 & -75.50 & -72.75 & -72.33 & -78.33 & -74.00 & -70.00 & -74.75 & -75.67 & -71.33 \\
$\Delta[\mathrm{dB}]$ & 3.75 & 4.00 & 3.58 & 2.92 & 3.83 & 2.83 & 3.33 & 4.50 & 2.75 & 3.58 & 4.33 & 4.00 & 3.25 & 5.00 & 3.67 & 4.33 \\
\hline
\end{tabular}

Table 2: Channel-specific RSSI variation of the $1 \mathrm{~h}$ average values of link $2 \rightarrow 4$ for one week (July 21-27, 2014)

\begin{tabular}{l|c|c|c|c|c|c|c|c|c|c|c|c|c|c|c|c||c}
\hline \multicolumn{1}{c}{ Ch } & 11 & 12 & 13 & 14 & 15 & 16 & 17 & 18 & 19 & 20 & 21 & 22 & 23 & 24 & 25 & 26 & Avg \\
PLE & & & & & & & & & & & & & & & & \\
\hline \hline$\mu$ & 2.07 & 2.03 & 2.03 & 2.04 & 2.10 & 2.01 & 2.05 & 2.13 & 2.18 & 2.05 & 2.12 & 2.15 & 2.16 & 2.12 & 2.17 & 2.14 & 2.10 \\
$\sigma$ & 0.25 & 0.19 & 0.14 & 0.25 & 0.23 & 0.16 & 0.16 & 0.22 & 0.32 & 0.17 & 0.23 & 0.27 & 0.31 & 0.20 & 0.24 & 0.20 & 0.22 \\
\hline $\max$ & 2.64 & 2.38 & 2.28 & 2.64 & 2.53 & 2.36 & 2.26 & 2.66 & 2.87 & 2.37 & 2.54 & 2.77 & 2.70 & 2.54 & 2.58 & 2.44 & 2.54 \\
$\min$ & 1.69 & 1.76 & 1.78 & 1.78 & 1.76 & 1.69 & 1.75 & 1.74 & 1.82 & 1.73 & 1.77 & 1.76 & 1.74 & 1.80 & 1.78 & 1.87 & 1.76 \\
$\Delta$ & 0.95 & 0.61 & 0.50 & 0.86 & 0.77 & 0.67 & 0.51 & 0.92 & 1.05 & 0.64 & 0.77 & 1.01 & 0.96 & 0.74 & 0.80 & 0.58 & 0.77 \\
\hline
\end{tabular}

Table 3: Inter-link PLE variation using a fixed channel based on all links' $(n=20)$ mean PLEs for one week (July 21-27, 2014).

\begin{tabular}{l|c|c|c}
\hline \multicolumn{1}{r}{ Technique } & E1 & E2 & E3 \\
PLE & link & link & link \\
\hline \hline$\mu$ & 2.10 & 1.90 & 1.92 \\
$\sigma$ & 0.19 & 0.14 & 0.14 \\
\hline $\max$ & 2.43 & 2.13 & 2.16 \\
$\min$ & 1.78 & 1.68 & 1.69 \\
$\Delta$ & 0.65 & 0.46 & 0.46 \\
\hline
\end{tabular}

Table 4: Inter-link PLE variation using adaptive link RSSI estimation based on all links' $(n=20)$ mean PLEs for one week (July 21-27, 2014).

descending order. We used the weights $w=\left\{w_{1}, w_{2}, w_{3}\right\}=$ $\left\{3 w_{3}, 2 w_{3}, w_{3}\right\}$, thus weighting the maximum value the most.

The results of these techniques are shown in Table 4, and the comparison with the fixed channels is illustrated in Figure 5. As can be seen, averaging the RSSI samples collected at different radio channels (ch $11 \ldots 26)$ for each link (E1) results in a smaller inter-link PLE variation than using a single fixed channel, on average. An even better way is to use the maximum RSSI value over the radio channels for each link (E2). In adaptive RSSI-based ranging, however, we apply the weighted mean over the best 3 RSSI values (E3), which results in a variation of the same size but is more robust.

Thereafter, to mitigate the node-specific variation of each two-way link between nodes $i$ and $j$, we simply average the RSSI values of the opposite directions as follows:

$$
R S S I_{1 \mathrm{~h}}=\frac{R S S I_{i j}+R S S I_{j i}}{2} .
$$

This compensates the differences (e.g., due to receiver noise, RF transceiver non-idealities) between the nodes. In particular, this could reduce the effect of different transmit powers on ranging results.

\subsection{Temperature Compensation}

The aim of temperature compensation is to mitigate the temporal intra-link RSSI/PLE variation caused by temperature variation. The effect of the temperature on RSSI can be estimated based on outdoor or laboratory measurements and using linear regression [8, 12, 13], for example. If estimated outdoors, RSSI and temperature data for a long-enough period are required in order that the temperature has time to change and for the results to be statistically significant. In controlled laboratory conditions, it is easier and quicker to estimate the effect, and the other potentially affecting factors can be ruled out. The estimated temperature effect can then be compensated in the ranging model. Temperature compensation $T C[\mathrm{~dB}]$ and temperature-compensated RSSI value $R S S I_{T C}$ at temperature $T_{i}\left[{ }^{\circ} \mathrm{C}\right]$ can be expressed as:

$$
\begin{gathered}
T C\left(T_{i}\right)=-\beta\left(T_{i}-T_{0}\right), \\
R S S I_{T C}=\operatorname{RSSI}\left(T_{i}\right)+T C\left(T_{i}\right),
\end{gathered}
$$

where $\beta$ is the coefficient of the temperature effect $\left[\mathrm{dB} /{ }^{\circ} \mathrm{C}\right]$ and $T_{0}$ the reference temperature. $T_{0}=25^{\circ} \mathrm{C}$ was chosen because it is the operating or ambient temperature used in the test conditions to define the technical parameters and specifications for AT86RF230 [30] and the electrical characteristics for Atmel ZigBit [29]. The effect of the temperature can be defined for the whole network or each link separately, by using a (i) WSNspecific $\beta$ or a (ii) link-specific $\beta$. In our evaluations, a WSNspecific $\beta$ is computed based on all the one-way reference links based on 1 week data. Use of a link-specific $\beta$ requires that the temperature sensors are integrated into each node. We use WSN-specific $\beta$ and temperatures, computed based on the averages of the reference links. We apply temperature compensation to the RSSI values of the reference nodes used in the PLE estimation and to the inter-node distance estimation by (unknown) nodes. An example of the effect of temperature compensation on the RSSI and PLE values for a link can be seen in Figure 6. The effect of temperature compensation on the intra-link PLE variation for each two-way link (link RSSI computed according to (E1)) can be seen in Table 5. As shown in the table, temperature compensation reduces the intra-link PLE variation for all the links. 


\begin{tabular}{l|l|l|l|l|l|l|l|l|l|l||l}
\hline \multicolumn{1}{c|}{ Link } & $1-2$ & $1-3$ & $1-4$ & $1-5$ & $2-3$ & $2-4$ & $2-5$ & $3-4$ & $3-5$ & $4-5$ & Avg \\
PLE & & & & & & & & & & & \\
\hline \hline$\mu$ & 2.06 & 1.92 & 1.92 & 1.78 & 2.33 & 2.13 & 2.00 & 2.16 & 2.42 & 2.23 & 2.10 \\
$\sigma$ & 0.06 & 0.05 & 0.04 & 0.04 & 0.05 & 0.03 & 0.04 & 0.05 & 0.04 & 0.06 & 0.05 \\
\hline$\mu_{\mathrm{TC}}$ & 2.06 & 1.93 & 1.92 & 1.78 & 2.34 & 2.13 & 2.00 & 2.17 & 2.42 & 2.24 & 2.10 \\
$\sigma_{\mathrm{TC}}$ & 0.01 & 0.01 & 0.01 & 0.01 & 0.03 & 0.02 & 0.01 & 0.02 & 0.02 & 0.02 & 0.02 \\
\hline
\end{tabular}

Table 5: Intra-link PLE variation (of (E1)) with and without temperature compensation (TC) for one week (July 21-27, 2014).

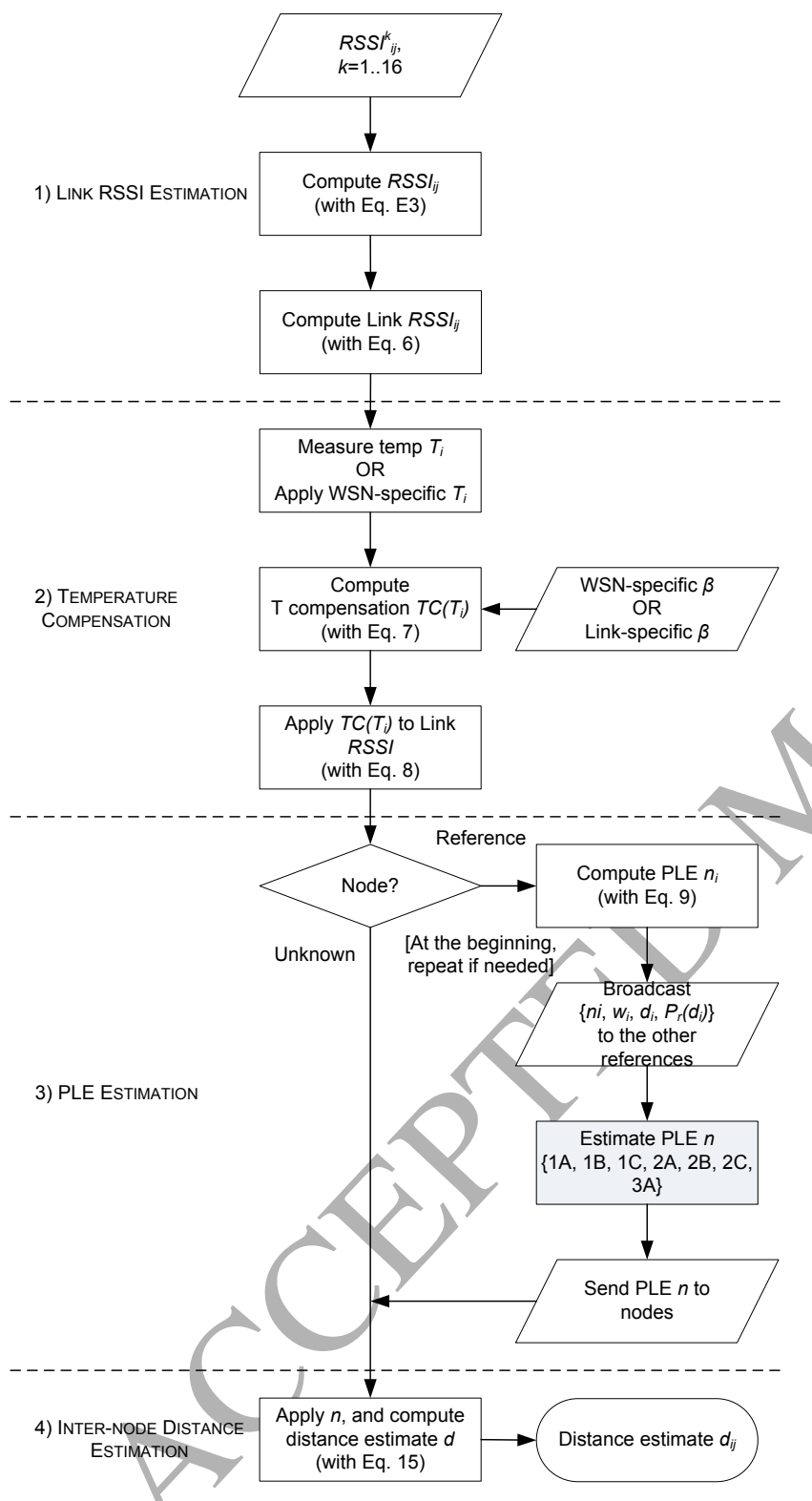

Figure 4: Adaptive RSSI-based ranging algorithm.

\subsection{PLE Estimation}

The aim of PLE estimation is to mitigate the error between the PLE applied in ranging and the true PLE for each link. A path loss exponent to be used in ranging is a key factor in determining the magnitude of the error. It is possible to apply either one (WSN-specific) or many (e.g., area-, node-, or link-

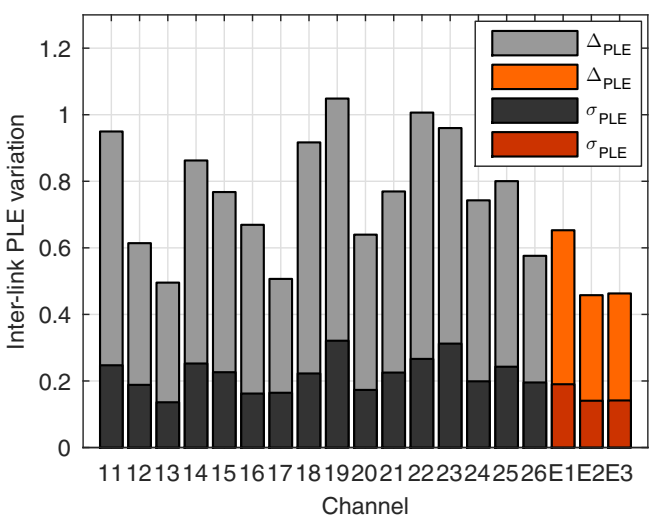

Figure 5: Inter-link PLE variation (combined from Table 3 and Table 4) based on all links' $(n=20)$ mean PLEs for one week (July 21-27, 2014).
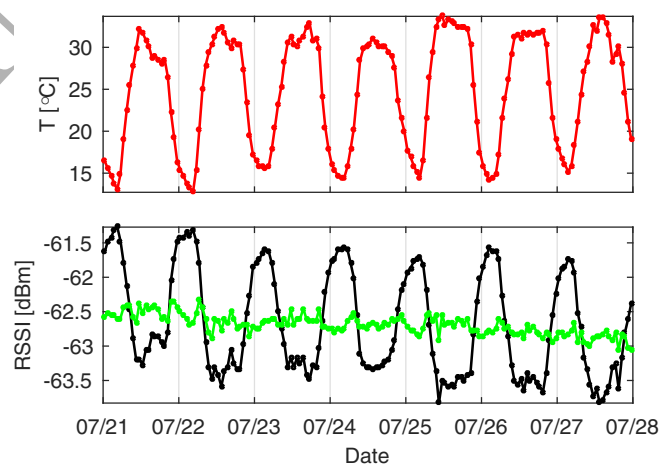

(a)

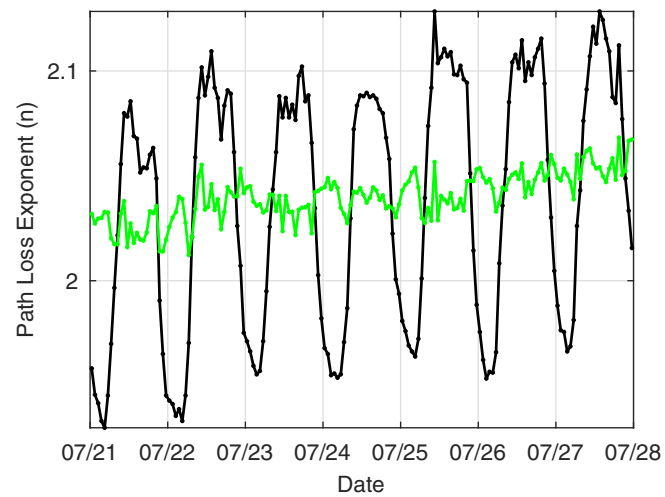

(b)

Figure 6: Effect of temperature and temperature compensation on (a) RSSI, (b) PLE for a link during 1 week (July 21-27, 2014). Temperature-compensated values shown in green. 


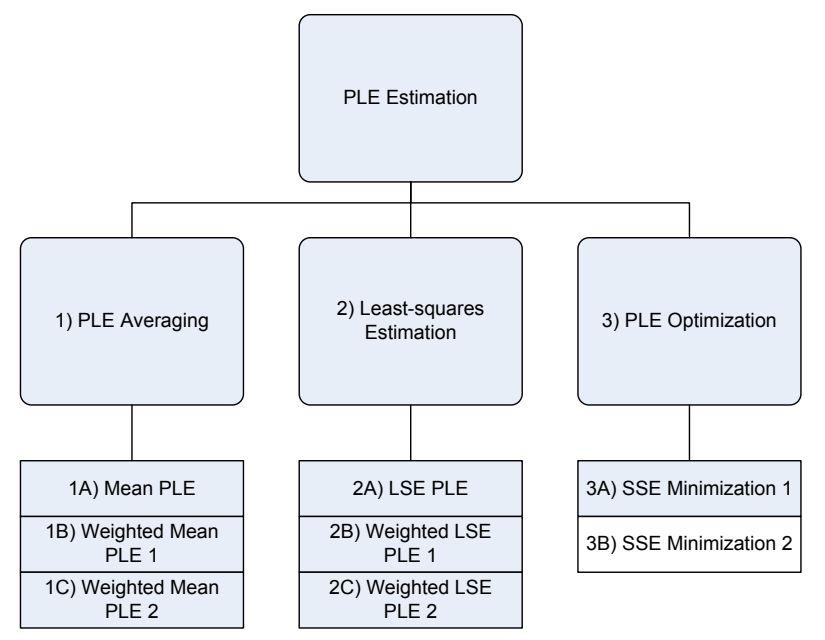

Figure 7: PLE estimation techniques.

specific) PLEs within a WSN. In this paper, we focus on the former method only due to its simplicity and due to our small test network.

Use of a predefined path loss exponent in RSSI-based ranging most likely results in an unnecessarily high error, i.e., low accuracy and precision. Nevertheless, the approximation of an appropriate PLE to be used in a particular environment in order to avoid large ranging errors is not a trivial task to accomplish. Use of a single PLE may result in large ranging errors, especially if the true PLE is higher than the estimated PLE, as shown previously.

A WSN-specific PLE can be estimated, e.g., by applying reference nodes and the known inter-node distances. The individual PLE $n_{i}$ of two-way reference link $i$ can be computed as

$$
n_{i}=\frac{\bar{P}_{r}\left(d_{i}\right)-P_{r}\left(d_{0}\right)}{-10 \log \left(d_{i} / d_{0}\right)}
$$

where $\bar{P}_{r}\left(d_{i}\right)$ is the average received power $[\mathrm{dBm}]$ of the link at distance $d_{i}$ based on the temperature-compensated RSSI measurements (e.g., over $24 \mathrm{~h}$ in this case). We compute the PLE only once, but it can be recomputed if necessary, e.g., on a daily basis.

Overall, the communication complexity of estimating the PLE is low. To estimate a WSN-specific PLE, the reference nodes broadcast the required data $\left(n_{i}, w_{i}, d_{i}, P_{r}\left(d_{i}\right)\right)$ to the other reference nodes only once (or infrequently at predefined intervals), and then send the computed estimate to the unknown nodes. The amount of communication depends on the number of reference nodes used to estimate the PLE. In all of the PLE estimation techniques, we use 3 reference nodes and links unless otherwise stated.

Next, we analyze different techniques to estimate a WSNspecific PLE based on reference links' PLEs to be used in ranging. They can be categorized as (i) PLE averaging, (ii) leastsquares estimation, and (iii) PLE optimization. These techniques are also illustrated in Figure 7.

\subsubsection{PLE Averaging}

A very straightforward technique for estimating a PLE is simply to compute the arithmetic mean PLE (1A) of the reference links $i=1 . . k$, i.e.,

$$
\hat{n}=\frac{1}{k} \sum_{i=1}^{k} n_{i}
$$

where $k$ is the number of reference links used for the computation (in our case, $k=3$ ).

The ordinary arithmetic mean does not account for the unequal effect of over- and underestimation of the PLE on ranging error, or the link distances. Therefore, we computed the weighted arithmetic mean PLE by applying different weighting methods as follows:

$$
\hat{n}=\frac{1}{W} \sum_{i=1}^{k} w_{i} n_{i}=\frac{1}{\sum_{i=1}^{k} w_{i}} \sum_{i=1}^{k} w_{i} n_{i},
$$

where $w_{i}$ is the weight for the PLE $n_{i}$.

In the first weighting technique (1B), the PLEs are weighted in the order of magnitude, i.e.,

$$
w_{i}= \begin{cases}\min w, & \text { if } n_{i}=\min n \\ : & \\ \max w, & \text { if } n_{i}=\max n .\end{cases}
$$

Thus, the bigger PLEs contribute more to the weighted mean as they produce smaller ranging errors. In our case, we used the weights $W=\left\{w(\min n)=w_{1}, w(\tilde{n})=2 w_{1}, w(\max n)=3 w_{1}\right\}$. However, they can be adjusted as needed.

In the second weighting technique (1C), the PLEs are weighted based on the relative ranging error when the mean PLE is used in ranging, i.e.,

$$
w_{i}=\frac{\left|\hat{d}_{i}-d_{i}\right|}{d_{i}},
$$

where $\hat{d}_{i}$ is the distance estimate for link $i$ when the mean PLE of the reference links (according to (10)) is used, and $d_{i}$ is the corresponding true distance. Thus, the PLE for a link whose true distance differs the most from the distance estimate using the mean PLE gets the biggest weight. In other words, we are trying to reduce the biggest error the most.

The computational complexity of PLE averaging is low. The computation required in these techniques can be easily performed in resource-constrained WSN reference nodes.

\subsubsection{Least-squares Estimation}

In a conventional PLE estimation, a standard procedure is to use ordinary least squares (OLS) estimation (2A) to solve $\arg \min _{\mathbf{n}} f(\mathbf{n})$, where $n$ is the PLE that minimizes the sum of the squared errors (residuals), i.e., vertical distances, between each sample and the corresponding point on the fitting curve, i.e.,

$$
\min f(n)=\min \sum_{i=1}^{k}\left(y_{i}-\hat{y}_{i}\right)^{2},
$$


where $y_{i}$ is the measured received power $P_{r}[\mathrm{dBm}]$ of the reference link $i$ and $\hat{y}_{i}$ is the corresponding $P_{r}$ on the fitting curve. Equation (12) can be expressed in matrix form as:

$$
\mathbf{A x}=\mathbf{b}
$$

where

$$
\mathbf{A}_{\mathbf{i}}=-10 \log \left(\frac{d_{i}}{d_{0}}\right), \mathbf{b}_{\mathbf{i}}=P_{r}\left(d_{i}\right)-P_{r}\left(d_{0}\right)
$$

for the reference link $i=1 . . k$. Solving the system of linear equations $\mathbf{A x}=\mathbf{b}$ for $\mathbf{x}$ (e.g., by using matrix factorization such as Cholesky or LU) gives the path loss exponent $\hat{n}$ which has the best fit to the given set of sample points.

The same problems as with the ordinary arithmetic mean apply for least squares. The least-squares estimate minimizes the error between the RSSI samples and the fit, which is not equivalent to minimizing the distance errors. By applying the weighted least-squares (WLS), it is possible to give different weights to each sample. In that case, $\hat{n}$ is the PLE that minimizes the sum of the weighted squared errors as

$$
\min f(n)=\min \sum_{i=1}^{k} w_{i}\left(y_{i}-\hat{y}_{i}\right)^{2}
$$

where $w_{i}$ is the weight for the reference link $i$. For the LSE, we use the same weighting techniques as for the mean PLE. In the first weighting technique (2B), the residuals are weighted in the order of magnitude of the corresponding PLEs, as in (11a) by using the same weights.

In the second weighting technique $(2 \mathrm{C})$, the residuals are weighted based on the relative ranging error when the leastsquares estimate PLE is used in ranging, i.e.,

$$
w_{i}=\frac{\left|\hat{d}_{i}-d_{i}\right|}{d_{i}}
$$

where $\hat{d}_{i}$ is the distance estimate for link $i$ when the $P L E_{L S E}$ of the reference links, i.e., $\arg \min _{\mathbf{n}} \sum_{i=1}^{k}\left(y_{i}-\hat{y}_{i}\right)^{2}$, is used, and $d_{i}$ is the corresponding true distance.

For computation, the complexity of the least-squares estimation techniques is higher than that of PLE averaging. This results mostly from solving the system of linear equations of modest size, defined by three reference nodes at least. In this case, however, it is fully practicable in WSN reference nodes as long as the number of references is small. The complexity of solving the system of linear equations is cubic of the problem dimensions (number of reference links), i.e., the total number of floating-point operations is $O\left(n^{3}\right)$. For example, if the number of reference nodes is 3 (5), the number of reference links is 3 (10), respectively. The actual implementation technique for solving the system of linear equations depends on the performance of the available sensor node's microcontroller.

\subsubsection{PLE Optimization}

In the sum of squared error (SSE) minimization, instead of weighting the reference node PLEs, we find the PLE between $\min _{i=1, k} n_{i}$ and $\max _{i=1, k} n_{i}$ for $k$ links that minimizes the overall distance estimate error (SSE) of the links used in the approximation. Specifically, we aim to solve the following optimization problem:

$$
\arg \min _{\hat{n}} f(\hat{n}):=\{\hat{n}, n \in S: f(\hat{n})=\min f(n)\} .
$$

In (14),

$$
\begin{aligned}
f(n)=\sum_{i=1}^{k} e_{i}^{2} & =\sum_{i=1}^{k}\left(\frac{\hat{d}_{i}-d_{i}}{d_{i}}\right)^{2} \\
& =\sum_{i=1}^{k}\left(\frac{d_{0} 10^{\left(P_{r}\left(d_{0}\right)-\widehat{P}_{r}\left(d_{i}\right)\right) / 10 n}-d_{i}}{d_{i}}\right)^{2},
\end{aligned}
$$

where $\hat{n}$ is the estimated PLE that minimizes $f(n), n$ is a PLE in $S, S \in\left[n_{\min }, n_{\max }\right]$ (with steps of 0.01 ), $k$ is the total number of two-way links with known distances used in the approximation, and $e_{i}$ is the relative distance estimate error of a two-way link $i$ between the estimated distance and the true distance, $\hat{d}_{i}$ and $d_{i}$, respectively. In [23], the authors apply a similar idea in a distributed fashion.

We computed the SSE minimization by using the reference links and all the links. In SSE Minimization 1 (3A), we performed the computation by using the reference links, i.e., $k=3$. As a baseline for evaluation, we also computed the SSE Minimization 2 (3B), where we used all the links, i.e., $k=10$. This is only for comparison to help evaluate the performance of the other techniques. It gives the minimum overall ranging error on average, when a single PLE is used in the ranging for all the links. In practice, however, it is not possible to compute a PLE for a link with an unknown distance by using equation (9).

The computational complexity of the SSE minimization techniques is also relatively low. In SSE Minimization 1 (3A), computation can be performed in WSN reference nodes. The complexity of solving the SSE minimization problem is linear of the problem dimensions (number of reference links), i.e., the total number of floating-point operations is $O(n)$. Naturally, SSE Minimization 2 (3B) cannot be implemented as unknown link distances are not known a priori in a real scenario.

\subsection{Inter-node Distance Estimation}

By applying the log-distance path loss model in (4) with link RSSI estimation, temperature compensation, and PLE estimation, the distance estimate $\hat{d}[\mathrm{~m}]$ between two nodes can be computed as follows:

$$
\hat{d}=d_{0} 10^{\left(P_{r}\left(d_{0}\right)-\bar{P}_{r, T C}(\hat{d})\right) / 10 \hat{n}},
$$

where $d_{0}$ is the reference distance $(1 \mathrm{~m}), P_{r}\left(d_{0}\right)$ is the received power at the reference distance $d_{0}, \bar{P}_{r, T C}(\hat{d})$ is the temperaturecompensated average received power $[\mathrm{dBm}]$ of the two-way link at distance $\hat{d}$ according to (8), and $\hat{n}$ is the estimated PLE according to the PLE estimation technique. In computing the $P_{r}\left(d_{0}\right)$, we used the Friis model (3) with parameters $P_{t}=-7.2 \mathrm{dBm}, G_{t}=G_{r}=5.5 \mathrm{dBi}, f=2442.5 \mathrm{MHz}$, and $L=1$. 


\subsection{Outline for WSN Implementation}

The following briefly outlines how the proposed algorithm could be implemented in WSN nodes. Here, we assume that nodes are similar to the one used in our experiment: (i) Nodes are operating at $2.4 \mathrm{GHz}$ band and (ii) integrated with temperature sensors (some or all). Also, (iii) the coefficient of temperature effect $(\beta)$ is known a priori. Overall, the aim is to reduce computation and communication without compromising ranging accuracy. Details of implementation are not in the scope of this paper.

The proposed algorithm runs on all nodes when it is necessary. The required data for the algorithm is collected by broadcasting at different radio channels in sequence. First, all nodes compute one-way link RSSI values by using the selected technique, and send/receive the RSSI values to/from neighbors for computing two-way link RSSI values. All nodes then use the latest temperature readings from reference nodes, and $\beta$ to compute temperature compensation for link RSSI. The PLE estimation phase runs only on reference nodes once at the beginning (e.g., based on 24 hours data). Each reference node computes PLEs of reference links between the neighboring reference nodes, and broadcast the PLEs and other required data to the other reference nodes. Then each reference node estimates a WSN-specific PLE by using one of the techniques described earlier. The computed WSN-specific PLE is then sent to other nodes. Finally, when a node needs to estimate inter-node distances, it applies the WSN-specific PLE and temperaturecompensated RSSI values in ranging.

All the PLE estimation techniques described are quite simple and can be implemented in our resource-constrained node (Atmel ZigBit $2.4 \mathrm{GHz}$ ), as long as the number of reference nodes is small. The PLE estimation phase can be repeated if necessary, e.g., on a daily basis. Admittedly, it is also possible to compute the WSN-specific PLE in unknown nodes instead of reference nodes if the reference nodes send the required data directly to the unknown nodes. This seems to be a potential option for implementation.

\section{Performance Evaluation of PLE Estimation Techniques}

The adaptive RSSI-based ranging algorithm was analyzed by using MATLAB [33]. To evaluate the performance of the adaptive RSSI-based ranging algorithm, we analyze and compare the ranging errors when we use different PLE estimation techniques, described in the previous section. The ranging errors analyzed here were computed for the transmit power level $\mathrm{P}_{\mathrm{TX} 2}$.

The quality of RSSI-based ranging was evaluated by analyzing the ranging accuracy and the precision. The average and standard deviation of the ranging error can be evaluated, e.g., by computing the mean absolute error (MAE), mean relative error (MRE), standard deviation of absolute error (SDAE), and standard deviation of relative error (SDRE) as follows:

$$
M A E=\frac{1}{n} \sum_{i=1}^{n}\left|\hat{d}_{i}-d_{i}\right|, M R E=\frac{1}{n} \sum_{i=1}^{n}\left|\frac{\hat{d}_{i}-d_{i}}{d_{i}}\right|,
$$

\begin{tabular}{c|c||c|c}
\hline Combination \# & Nodes & Combination \# & Nodes \\
\hline 1 & $\{1,2,3\}$ & 6 & $\{1,4,5\}$ \\
2 & $\{1,2,4\}$ & 7 & $\{2,3,4\}$ \\
3 & $\{1,2,5\}$ & 8 & $\{2,3,5\}$ \\
4 & $\{1,3,4\}$ & 9 & $\{2,4,5\}$ \\
5 & $\{1,3,5\}$ & 10 & $\{3,4,5\}$ \\
\hline
\end{tabular}

Table 6: Reference node combinations.

$$
S D A E / S D R E=\sqrt{\frac{1}{n} \sum_{i=1}^{n}\left(e_{i}-\bar{e}\right)^{2}},
$$

where $n$ is the number of $1 \mathrm{~h}$ samples $i$ for a two-way link during the measurement period ( 7 days), $\hat{d}_{i}$ is the distance estimate, $d_{i}$ is the true distance, $e_{i}$ is the ranging error (absolute or relative) of sample $i$, and $\bar{e}$ is the average error (absolute or relative) of the samples. The links were classified based on the inter-node distances as short $(15-30 \mathrm{~m})$, medium (40-55 m), and long (65-95 m) links. The average MAE/MRE and SDAE/SDRE were computed for each class, and for the unknown (excluding the reference links) and all the links. This procedure was conducted for each 3-combination of the reference nodes $(k=3)$ from a set of 5 nodes $(n=5)$, i.e., for $\left(\begin{array}{l}n \\ k\end{array}\right)=10$ combinations, shown in Table 6. As a result, the final MAE/MRE and SDAE/SDRE values used in the evaluation were computed by averaging over the mean values of these 10 combinations. First, we analyze the results of the different averaging, LSE, and optimization techniques for the PLE separately. Then, we compare them with each other.

\subsection{PLE Averaging}

The results presenting the relative and absolute ranging errors of the different PLE averaging techniques can be found in Table 7. As can be seen, in this scenario, weighted mean PLE 1 (1B) is the best technique for nearly every class. Similarly, the second best technique for every class is the weighted mean PLE 2 (1C). This indicates that it is sensible to use the weighted mean instead of the simple arithmetic mean (1A), preferable by weighting the PLEs in the order of magnitude. The only exception is the MRE/MAE for long links, in which case the simple mean produces the smallest ranging error. One possible explanation for this might be that the PLEs of the long links are, in this case, mostly smaller than those of the shorter ones. If the bigger PLEs are emphasized more in estimating the PLE, then the difference between the long links' PLEs and the estimated PLE increases more than compared to the mean PLE. Further, the PLE error is emphasized for longer distances. With all the techniques, the SDRE is smallest for the long links, while the MRE/MAE is smallest for the short links.

\subsection{Least-squares Estimation}

The results presenting the relative and absolute ranging errors of the different PLE least-squares estimation techniques can be found in Table 8. As can be seen, in this scenario, weighted LSE PLE 1 (2B) is the best technique for nearly every class. The only exception is the MRE/MAE of the long links, in which 


\begin{tabular}{|c|c|c|c|c|c|c|c|c|c|c|}
\hline $\begin{array}{l}\text { PLE Estimation Technique } \\
\text { Short links }=15-30 \mathrm{~m} \\
\text { Medium links }=40-55 \mathrm{~m} \\
\text { Long links }=65-95 \mathrm{~m}\end{array}$ & $\begin{array}{l}\text { Mean } \\
\text { Short } \\
\text { links } \\
(4)\end{array}$ & $\begin{array}{l}\text { Mean } \\
\text { Medium } \\
\text { links } \\
(3)\end{array}$ & $\begin{array}{l}\text { Mean } \\
\text { Long } \\
\text { links } \\
(3)\end{array}$ & $\begin{array}{c}\text { Mean } \\
\text { Unk. } \\
\text { links } \\
(7)\end{array}$ & $\begin{array}{l}\text { Mean } \\
\text { All } \\
\text { links } \\
(10)\end{array}$ & $\begin{array}{l}\text { SD } \\
\text { Short } \\
\text { links } \\
(4)\end{array}$ & $\begin{array}{c}\text { SD } \\
\text { Medium } \\
\text { links } \\
(3)\end{array}$ & $\begin{array}{l}\text { SD } \\
\text { Long } \\
\text { links } \\
(3)\end{array}$ & $\begin{array}{c}\text { SD } \\
\text { Unk. } \\
\text { links } \\
(7) \\
\end{array}$ & $\begin{array}{c}\text { SD } \\
\text { All } \\
\text { links } \\
(10)\end{array}$ \\
\hline $\begin{array}{l}\text { 1A) Mean PLE } \\
\text { Relative rangin } \\
\text { Absolute rangin }\end{array}$ & $\begin{array}{c}0.224 \\
5.35\end{array}$ & & & & & $\begin{array}{c}0.055 \\
1.34\end{array}$ & $\begin{array}{c}0.060 \\
2.92\end{array}$ & $\begin{array}{c}0.028 \\
2.18\end{array}$ & $\begin{array}{c}0.051 \\
2.14\end{array}$ & $\begin{array}{c}0.049 \\
2.06\end{array}$ \\
\hline $\begin{array}{r}\text { 1B) We } \\
\text { Relat } \\
\text { Abso }\end{array}$ & $\begin{array}{c}0.180 \\
4.24 \\
\end{array}$ & & & & $\begin{array}{l}0.260 \\
14.18 \\
\end{array}$ & $\begin{array}{c}0.050 \\
1.22 \\
\end{array}$ & & $\begin{array}{r}0.025 \\
1.95 \\
\end{array}$ & $\begin{array}{c}0.046 \\
1.93 \\
\end{array}$ & $\begin{array}{c}0.044 \\
1.86 \\
\end{array}$ \\
\hline $\begin{array}{l}\text { 1C) Weighted Mean PLE } 2 \\
\text { Relative ranging error }[\times d] \\
\text { Absolute ranging error }[\mathrm{m}]\end{array}$ & $\begin{array}{c}0.208 \\
4.92\end{array}$ & $\begin{array}{l}0.310 \\
15.28\end{array}$ & $\begin{array}{l}0.310 \\
25.12\end{array}$ & $\begin{array}{l}0.300 \\
15.68 \\
\end{array}$ & $\begin{array}{l}0.269 \\
14.09 \\
\end{array}$ & $\begin{array}{c}0.054 \\
1.31 \\
\end{array}$ & $\begin{array}{c}0.058 \\
2.83\end{array}$ & $\begin{array}{c}0.027 \\
2.11\end{array}$ & $\begin{array}{c}0.050 \\
2.08 \\
\end{array}$ & $\begin{array}{c}0.047 \\
2.01\end{array}$ \\
\hline
\end{tabular}

Table 7: Average ranging errors of PLE averaging techniques based on all the reference nodes' 3-combinations (10). A measurement period of one week (July 21-27, 2014).

\begin{tabular}{|c|c|c|c|c|c|c|c|c|c|c|}
\hline $\begin{array}{l}\text { PLE Estimation Technique } \\
\text { Short links }=15-30 \mathrm{~m} \\
\text { Medium links }=40-55 \mathrm{~m} \\
\text { Long links }=65-95 \mathrm{~m}\end{array}$ & $\begin{array}{c}\text { Mean } \\
\text { Short } \\
\text { links } \\
(4)\end{array}$ & $\begin{array}{l}\text { Mean } \\
\text { Medium } \\
\text { links } \\
(3)\end{array}$ & $\begin{array}{c}\text { Mean } \\
\text { Long } \\
\text { links } \\
(3)\end{array}$ & $\begin{array}{l}\text { Mean } \\
\text { Unk. } \\
\text { links } \\
(7)\end{array}$ & $\begin{array}{c}\text { Mean } \\
\text { All } \\
\text { links } \\
(10) \\
\end{array}$ & $\begin{array}{l}\text { SD } \\
\text { Short } \\
\text { links } \\
(4)\end{array}$ & $\begin{array}{l}\text { SD } \\
\text { Medium } \\
\text { links } \\
(3)\end{array}$ & $\begin{array}{l}\text { SD } \\
\text { Long } \\
\text { links } \\
(3) \\
\end{array}$ & $\begin{array}{l}\text { SD } \\
\text { Unk. } \\
\text { links } \\
(7)\end{array}$ & $\begin{array}{c}\text { SD } \\
\text { All } \\
\text { links } \\
(10) \\
\end{array}$ \\
\hline \multicolumn{11}{|l|}{ 2A) LSE PLE } \\
\hline & .252 & 346 & 0.273 & 0.3 & 0.286 & 0.058 & 0.063 & 0.030 & 0.053 & 0.051 \\
\hline Absol & 6. & & 2.25 & & & 1.40 & 3.06 & 2.28 & 2.24 & 2.16 \\
\hline \multicolumn{11}{|l|}{ 2B) Weighted LSE PLE 1} \\
\hline & $.1{ }^{1}$ & ר) & 0.330 & 0.3 & 0.267 & 0.052 & 0.056 & 0.026 & 0.048 & 0.045 \\
\hline & & & & & 14.22 & 1.26 & 2.71 & 2.03 & 2.00 & 1.92 \\
\hline \multicolumn{11}{|l|}{ 2C) Weighted LSE PLE 2} \\
\hline & 2 & & 0.308 & 0.3 & 0.273 & 0.055 & 0.059 & 0.028 & 0.050 & 0.048 \\
\hline Absolute ranging error $[\mathrm{m}]$ & 5.09 & 15.64 & 24.93 & 15,90 & 14.21 & 1.32 & 2.86 & 2.14 & 2.10 & 2.03 \\
\hline
\end{tabular}

Table 8: Average ranging errors of LSE techniques based on all the reference nodes' 3-combinations (10). A measurement period of one week (July 21-27, 2014).

case the simple LSE PLE (2A) produces the smallest ranging error, probably due to the same reason as explained above. The second best technique for every class is the weighted LSE PLE 2 (2C). For the LSE technique, too, it seems that using weighted LSE, especially LSE PLE 1 (2B), yields a smaller ranging error than simple LSE (2A) in generat. With all the techniques, the SDRE is smallest for the long links, while the MRE/MAE is smallest for the short links.

\subsection{PLE Optimization}

The results for the relative and absolute ranging errors of the PLE optimization techniques can be found in Table 9. We compare SSE Minimization 1 (3A) with SSE Minimization 2 (3B), which is computed the same way by using all the links, not just the reference links. As can be seen, the SSE Minimization 2 technique outperforms the SSE Minimization 1 technique, which was presumable. However, SSE Minimization 1 is better for long links. The ranging errors for the different length links are in line with the previous techniques.

\subsection{Comparison between Techniques}

We chose the best PLE averaging (1B) and least-squares estimation (2B) techniques and compared them with the SSE Minimization 1 (3A) and SSE Minimization 2 (3B) techniques. The results are shown in Table 9. As can be seen, in this scenario, the best technique altogether is weighted mean PLE 1 (1B) (apart from 3B). It yields the smallest ranging error for every class except the MRE/MAE for long links, in which case weighted LSE PLE 1 (2B) is the best. They both outperform the SSE Minimization 1 (3A) technique in nearly every case. Weighting the bigger PLEs more seems to result in better outcomes in general, for the averaging and LSE methods. This illustrates that optimizing the PLE based on the reference links does not necessarily result in the best outcome for unknown links, particularly if their PLEs differ considerably from those of the reference links. The results of the techniques are actually quite close to the baseline, SSE Minimization 2 (3B). This manifests the challenges of using a single PLE in ranging.

Although the MRE/MAE and SDRE/SDAE values averaged over the links and combinations give a good idea of the performance of the techniques in general, they do not tell anything about how the error is distributed between different links and reference node combinations. Therefore, we computed the MRE and SDRE values for each link and reference node combination separately. They are depicted in Figure 8 and Figure 9, respectively. As can be seen, the MRE and the SDRE vary quite heavily between the links. One link in particular $(3-5)$ 


\begin{tabular}{|c|c|c|c|c|c|c|c|c|c|c|}
\hline $\begin{array}{l}\text { PLE Estimation Technique } \\
\text { Short links = } 15-30 \mathrm{~m} \\
\text { Medium links }=40-55 \mathrm{~m} \\
\text { Long links }=65-95 \mathrm{~m}\end{array}$ & $\begin{array}{c}\text { Mean } \\
\text { Short } \\
\text { links } \\
(4)\end{array}$ & $\begin{array}{l}\text { Mean } \\
\text { Medium } \\
\text { links } \\
(3) \\
\end{array}$ & $\begin{array}{c}\text { Mean } \\
\text { Long } \\
\text { links } \\
(3)\end{array}$ & $\begin{array}{l}\text { Mean } \\
\text { Unk. } \\
\text { links } \\
(7)\end{array}$ & $\begin{array}{c}\text { Mean } \\
\text { All } \\
\text { links } \\
(10)\end{array}$ & $\begin{array}{l}\text { SD } \\
\text { Short } \\
\text { links } \\
(4)\end{array}$ & $\begin{array}{c}\text { SD } \\
\text { Medium } \\
\text { links } \\
(3) \\
\end{array}$ & $\begin{array}{l}\text { SD } \\
\text { Long } \\
\text { links } \\
(3)\end{array}$ & $\begin{array}{c}\text { SD } \\
\text { Unk. } \\
\text { links } \\
(7) \\
\end{array}$ & $\begin{array}{c}\text { SD } \\
\text { All } \\
\text { links } \\
(10)\end{array}$ \\
\hline 1B) Weighted Mean PLE 1 & & & & & & & & & & \\
\hline & & & & & & $\begin{array}{c}0.050 \\
1.22\end{array}$ & $\begin{array}{c}0.054 \\
2.61\end{array}$ & $\begin{array}{c}0.025 \\
1.95\end{array}$ & $\begin{array}{c}0.046 \\
1.93\end{array}$ & $\begin{array}{c}0.044 \\
1.86\end{array}$ \\
\hline 2B) $\mathrm{We}$ & & & & & & & & & & \\
\hline & $\begin{array}{c}0.198 \\
4.68\end{array}$ & & $\begin{array}{l}0.330 \\
26.69\end{array}$ & $\begin{array}{l}0.303 \\
16.02\end{array}$ & & $\begin{array}{c}0.052 \\
1.26\end{array}$ & $\begin{array}{c}0.056 \\
2.71\end{array}$ & $\begin{array}{c}0.026 \\
2.03\end{array}$ & $\begin{array}{c}0.048 \\
2.00\end{array}$ & $\begin{array}{c}0.045 \\
1.92\end{array}$ \\
\hline $\begin{array}{l}\text { 3A) SSE Minimization 1 } \\
\text { Relative ranging error }[\times d] \\
\text { Absolute ranging error }[\mathrm{m}]\end{array}$ & $\begin{array}{c}0.196 \\
4.64\end{array}$ & $\begin{array}{l}0.298 \\
14.56\end{array}$ & $\begin{array}{l}0.334 \\
27.03\end{array}$ & $\begin{array}{l}0.306 \\
16.18\end{array}$ & $\begin{array}{l}0.268 \\
14.33\end{array}$ & $\begin{array}{c}0.052 \\
1.25\end{array}$ & $\begin{array}{c}0.055 \\
2.69\end{array}$ & & $\begin{array}{l}0.048 \\
1.99\end{array}$ & $\begin{array}{c}0.045 \\
1.91\end{array}$ \\
\hline $\begin{array}{l}\text { 3B) SSE Minimization } 2 \\
\text { Relative ranging error }[\times d] \\
\text { Absolute ranging error }[\mathrm{m}]\end{array}$ & $\begin{array}{c}0.125 \\
2.83\end{array}$ & $\begin{array}{l}0.243 \\
11.63\end{array}$ & $\begin{array}{l}0.382 \\
30.77\end{array}$ & $\begin{array}{l}0.237 \\
13.84\end{array}$ & $\begin{array}{l}0.237 \\
13.85\end{array}$ & $\begin{array}{c}0.047 \\
1.14\end{array}$ & & $\begin{array}{c}0.024 \\
1.81\end{array}$ & $\begin{array}{c}0.042 \\
1.76\end{array}$ & $\begin{array}{c}0.041 \\
1.72\end{array}$ \\
\hline
\end{tabular}

Table 9: Average ranging errors of PLE estimation techniques based on all the reference nodes' 3-combinations (10). A measurement period of one week (July 21-27, 2014).

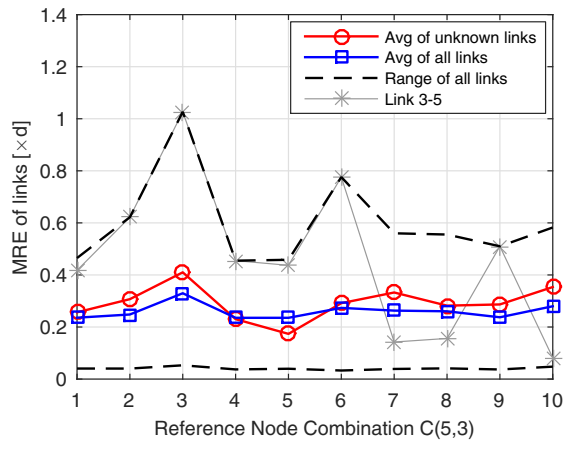

(a)

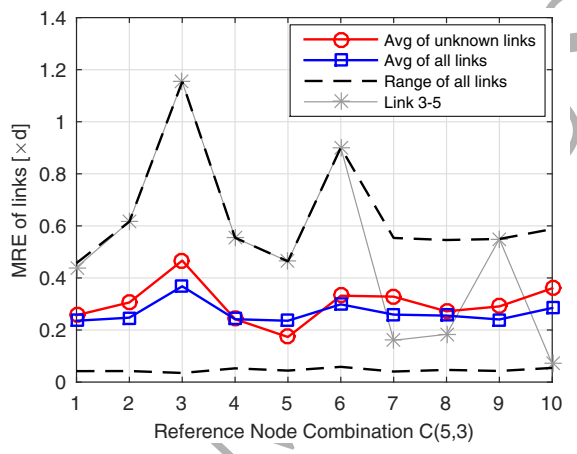

(b)

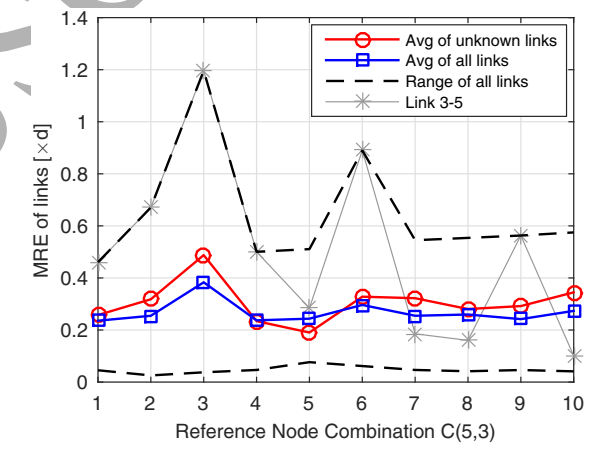

(c)

Figure 8: MRE of the links vs. reference node combinations (see Table 6) using the PLE estimation technique (a) Weighted Mean PLE 1, (b) Weighted LSE PLE 1, (c) SSE Minimization 1. A measurement period of one week (July 21-27, 2014).

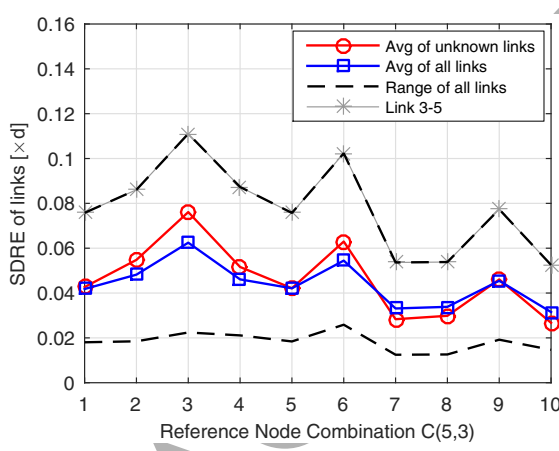

(a)

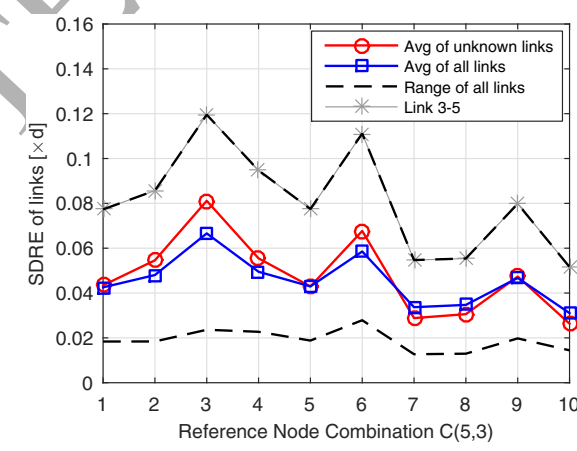

(b)

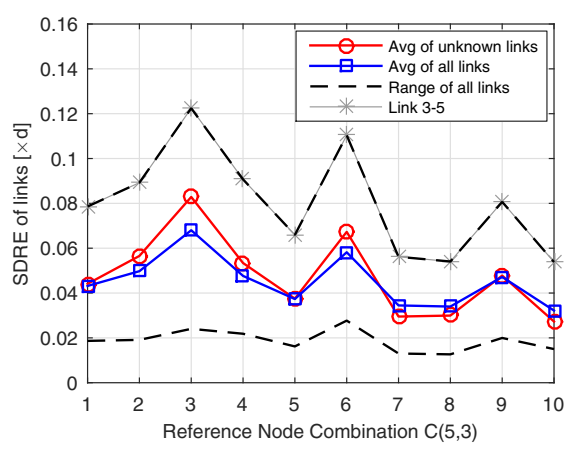

(c)

Figure 9: SDRE of the links vs. reference node combinations (see Table 6) using the PLE estimation technique (a) Weighted Mean PLE 1, (b) Weighted LSE PLE 1, (c) SSE Minimization 1. A measurement period of one week (July 21-27, 2014).

clearly has a bigger MRE than the others in most cases. Furthermore, the MRE and the SDRE vary depending on the reference nodes. In the figures, we can clearly identify two or three bad reference node combinations that appear as high peaks. These are combinations $3=\{1,2,5\}, 6=\{1,4,5\}$, and $9=\{2,4,5\}$. The results indicate that link and reference node selection play an important role in ranging and location accuracy. The differences between the PLE estimation techniques, however, are quite marginal.

In conclusion, the ranging accuracy of the analyzed PLE estimation techniques is reasonable, on average, but varies considerably depending on the link. In any case, use of a single 
WSN-specific PLE will not result in highly accurate ranging due to the inherent variation of PLEs between links, not even in a small geographic area as in our case where the links are in the same direction.

\section{Discussion}

Our findings demonstrate the negative effects of PLE estimation error and temperature change on RSSI-based ranging. Clearly, there is a need for some kind of error mitigation techniques. This paper aimed to find a cost-effective solution to the given problem. The proposed adaptive method is relatively low-cost and simple, and it can be implemented in resource-constrained sensor nodes. The algorithm integrates link RSSI estimation, temperature compensation, and PLE estimation techniques with inter-node distance estimation to improve the range quality.

The results of this study indicate that employing the most appropriate channels relative to the ranging for each link reduces the inter-link PLE variation compared to the use of a single fixed channel. Consequently, the ranging error is reduced. Taking the weighted mean of the best RSSI values for each link at a particular time seems to be a sensible choice. Choosing the maximum RSSI also results in comparable outcomes, but it is less robust. These findings suggest that an appropriate linkRSSI estimation should be included as a part of RSSI-based ranging.

Another important finding was that the positive effect of temperature compensation on ranging is clear. Temperature compensation reduces the temporal RSSI variation, and therefore, the intra-link PLE variation. This also results in a smaller variation in the ranging error. If the PLE is given, or computed infrequently, temperature compensation is necessary to ranging accuracy/precision. Alternatively, temperature compensation will not be necessary if the PLE is computed every time when a node estimates distances. However, temperature compensation is cost-effective and, therefore, the preferred option.

The differences between the PLE estimation techniques were quite marginal, although one method was slightly better than the others. Therefore, it is not that relevant to ranging accuracy which technique to use. In that case, the PLE estimation technique should be selected mainly based on its computational complexity. In contrast, the differences in the ranging accuracy and precision were considerable depending on which link and reference nodes were in question.

One interesting result is that the MRE of the short links is the smallest. That results from the fact that the PLE error has a lower impact on the ranging error for short distances, as was shown previously (this is emphasized even more if the errors are presented in meters). Therefore, it might be reasonable to favor shorter links in localization instead of longer ones for finding the shortest paths between unknown and reference nodes. Furthermore, in large WSNs or those with diverse propagation environments, it might be sensible to divide the network into smaller areas to apply the PLE estimation. This would reduce the amount of communication and would probably result in better PLE estimates.
Overall, the results are promising but show the challenges related to RSSI-based ranging. If the inter-link PLE variation is not considerable, WSN-specific PLE estimation yields better results. However, if there are large deviations in the PLEs between the links, the ranging errors could be considerable. Although link RSSI estimation and temperature compensation can mitigate ranging errors to a certain extent, these techniques cannot remove the general problem that arises when a single WSNspecific PLE is used in ranging in the presence of high inter-link PLE variation. Furthermore, the reference links' PLEs used for computing the WSN-specific PLE may differ considerably from those of unknown links. Therefore, alternative methods are needed. One potential and interesting approach is to estimate a specific PLE for each link with some technique. However, estimating link-specific PLEs for ranging cost-effectively requires further studies and remains as future work.

\section{Conclusion}

This paper set out to improve RSSI-based ranging quality in WSNs by adapting to changing outdoor conditions. We showed the effects of path loss exponent estimation error and temperature change on RSSI-based ranging, and how to mitigate them using adaptive techniques. Furthermore, we evaluated the proposed adaptive RSSI-based ranging algorithm with different PLE estimation techniques. The results indicate that although adaptive techniques could reduce the ranging error, the accuracy achieved when a single PLE estimate is used varies considerably depending on the link and the reference nodes due to the high inter-link PLE variation. The results of this study are particularly useful when employing RSSI-based ranging and localization for WSNs in outdoor conditions, but they can also be applied on a larger scale.

\section{References}

\section{References}

[1] W. Dargie, C. Poellabauer, Fundamentals of Wireless Sensor Networks: Theory and Practice, Wiley Series on Wireless Communications and Mobile Computing, John Wiley \& Sons Ltd., 2010.

[2] H. Karl, A. Willig, Protocols and Architectures for Wireless Sensor Networks, John Wiley \& Sons Ltd., 2006.

[3] I. Akyildiz, W. Su, Y. Sankarasubramaniam, E. Cayirci, Wireless sensor networks: a survey, Computer Networks 38 (4) (2002) 393-422, https : //doi.org/10.1016/S1389-1286(01)00302-4.

[4] N. Patwari, et al., Locating the Nodes: Cooperative localization in wireless sensor networks, IEEE Signal Processing Magazine 22 (4) (2005) 54-69, https ://doi.org/10.1109/MSP. 2005.1458287.

[5] A. Boukerche, H. Oliveira, E. Nakamura, A. Loureiro, Localization Systems for Wireless Sensor Networks, IEEE Wireless Communications 14 (6) (2007) 6-12, https://doi.org/10.1109/MWC. 2007. 4407221.

[6] G. Mao, B. Fidan, B. Anderson, Wireless sensor network localization techniques, Computer Networks 51 (10) (2007) 2529-2553, https:// doi.org/10.1016/j. comnet.2006.11.018.

[7] J. Zhao, et al., Localization of Wireless Sensor Networks in the Wild: Pursuit of Ranging Quality, IEEE/ACM Transactions on Networking 21 (1) (2013) 311-323, https ://doi .org/10.1109/TNET . 2012.2200906. 
[8] K. Bannister, G. Giorgetti, S. Gupta, Wireless Sensor Networking for "Hot" Applications: Effects of Temperature on Signal Strength, Data Collection and Localization, in: The Fifth Workshop on Embedded Networked Sensors (HotEmNets'08), Charlottesville, Virginia, USA, 2008.

[9] C. Boano, J. Brown, Z. He, U. Roedig, T. Voigt, Low-Power Radio Communication in Industrial Outdoor Deployments: The Impact of Weather Conditions and ATEX-Compliance, in: N. Komninos (Ed.), Sensor Applications, Experimentation, and Logistics: First International Conference, SENSAPPEAL 2009, Vol. 29 of LNICST, Springer, Athens, Greece, 2009, pp. 159-176, https://doi.org/10.1007/ 978-3-642-11870-8_11.

[10] J. Luo, X. Xu, Q. Zhang, Understanding Link Feature of Wireless Sensor Networks in Outdoor Space: a Measurement Study, in: IEEE Global Telecommunications Conference (GLOBECOM 2011), Houston, TX, USA, 2011, https://doi.org/10.1109/GLOCOM.2011.6134117.

[11] H. Wennerström, F. Hermans, O. Rensfelt, C. Rohner, L. Nordén, A Long-Term Study of Correlations between Meteorological Conditions and 802.15.4 Link Performance, in: 2013 IEEE International Conference on Sensing, Communications and Networking (SECON), New Orleans, LA, USA, 2013, pp. 221-229, https://doi.org/10.1109/SAHCN . 2013.6644981.

[12] C. Boano, et al., Hot Packets: A Systematic Evaluation of the Effect of Temperature on Low Power Wireless Transceivers, in: 5th Extreme Conference on Communication (ExtremeCom'13), Thorsmork, Iceland, 2013.

[13] J. Luomala, I. Hakala, Effects of Temperature and Humidity on Radio Signal Strength in Outdoor Wireless Sensor Networks, in: M. Ganzha, L. Maciaszek, M. Paprzycki (Eds.), Proceedings of the 2015 Federated Conference on Computer Science and Information Systems (FedCSIS 2015), Vol. 5 of ACSIS, 2015, pp. 1247-1255, https://doi.org/10. 15439/2015F241.

[14] A. Awad, T. Frunzke, F. Dressler, Adaptive Distance Estimation and Localization in WSN using RSSI Measures, in: 10th Euromicro Conference on Digital System Design Architectures, Methods and Tools (DSD 2007), 2007, pp. 471-478, https://doi.org/10.1109/DSD. 2007. 4341511.

[15] J. Xu, W. Liu, F. Lang, Y. Zhang, C. Wang, Distance Measurement Model Based on RSSI in WSN, Wireless Sensor Network 2 (2010) 606-611, https://doi.org/10.4236/wsn.2010.28072.

[16] M. Botta, M. Simek, Adaptive Distance Estimation Based on RSSI in 802.15.4 Network, Radioengineering 22 (4) (2013) 1162-1168.

[17] G. Mao, B. D.O. Anderson, B. Fidan, Path loss exponent estimation for wireless sensor network localization, Computer Networks 51 (10) (2007) 2467-2483, https://doi.org/10.1016/j.comnet.2006.11.007.

[18] S. Srinivasa, M. Haenggi, Path Loss Exponent Estimation in Large Wireless Networks, in: Information Theory and Applications Workshop, San Diego, CA, USA, 2009, pp. 124-129, https://doi.org/10.1109/ ITA. 2009.5044933.

[19] Y. Hu, G. Leus, Self-Estimation of Path-Loss Exponent in Wireless Networks and Applications, IEEE Transactions on Vehicular Technology 64 (11) (2015) 5091-5102, https://doi.org/10.1109/TVT. 2014. 2380823.

[20] Y. Hu, G. Leus, Directional Maximum Likelihood Self-Estimation of the Path-Loss Exponent, in: IEEE International Conference on Acoustics, Speech and Signal Processing (ICASSP), Shanghai, China, 2016, pp. 3806-3810, https ://doi .org/10.1109/ICASSP. 2016.7472389.

[21] J.-H. Choi, J.-K. Choi, S.-J. Yoo, Iterative Path-Loss Exponent Estimation-based Positioning Scheme in WSNs, in: 4th International Conference on Ubiquitous and Future Networks (ICUFN), Phuket, Thailand, 2012, pp. 23-26, https://doi.org/10.1109/ICUFN.2012. 6261658.

[22] G. Wang, H. Chen, Y. Li, M. Jin, On Received-Signal-Strength Based Localization with Unknown Transmit Power and Path Loss Exponent, IEEE Wireless Communications Letters 1 (5) (2012) 536-539, https: //doi.org/10.1109/WCL. 2012.072012.120428.

[23] A. Bel, J. L. Vicario, G. Seco-Granados, Localization Algorithm with On-line Path Loss Estimation and Node Selection, Sensors 11 (7) (2011) 6905-6925, https://doi.org/10.3390/s110706905.

[24] C. C. Pu, S. Y. Lim, P. C. Ooi, Measurement Arrangement for the Estimation of Path Loss Exponent in Wireless Sensor Network, in: 7th International Conference on Computing and Convergence Technology (ICCCT),
Seoul, South Korea, 2012, pp. 807-812.

[25] Y. Huang, J. Zheng, Y. Xiao, M. Peng, Robust Localization Algorithm Based on the RSSI Ranging Scope, International Journal of Distributed Sensor Networks 11 (2) (2015), https://doi.org/10.1155/2015/ 587318.

[26] A. Zanella, A. Bardella, RSS-Based Ranging by Multichannel RSS Averaging, IEEE Wireless Communications Letters 3 (1) (2014) 10-13, https://doi.org/10.1109/WCL. 2013.100913.130631.

[27] G. Betta, D. Capriglione, D. Casinelli, L. Ferrigno, Experimental Analysis of the Frequency Diversity to Improve Localization in WSNs, in: XVIII AISEM Annual Conference, Trento, Italy, 2015, https://doi. org/10.1109/AISEM. 2015.7066820.

[28] T. Rappaport, Wireless Communications: Principles and Practice, 2nd Edition, Prentice Hall PTR, 2001.

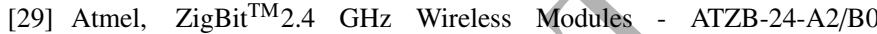
Datasheet, http: //www . atmel.com (2009).

[30] Atmel, Low Power 2.4 GHz Transceiver for ZigBee, IEEE 802.15.4, 6LoWPAN, RF4CE and ISM Applications - AT86RF230 Datasheet, http: //www . atmel . com (2009).

[31] Sensirion, Datasheet SHT7x (SHT71, SHT75) - Humidity and Temperature Sensor IC, Version 5, http://www. sensirion.com (2011).

[32] A. Bardella, N. Bui, A. Zanella, M. Zorzi, An Experimental Study on IEEE 802.15.4 Multichannel Transmission to Improve RSSIBased Service Performance, in: 4th International Workshop on RealWorld Wireless Sensor Networks (REALWSN 2010), LNCS 6511, Colombo, Sri Lanka, 2010, pp. 154-161, https : //doi .org/10.1007/ 978-3-642-17520-6_15.

[33] The MathWorks, Inc., MATLAB - The Language of Technical Computing, http://www . mathworks.se/products/matlab/. 


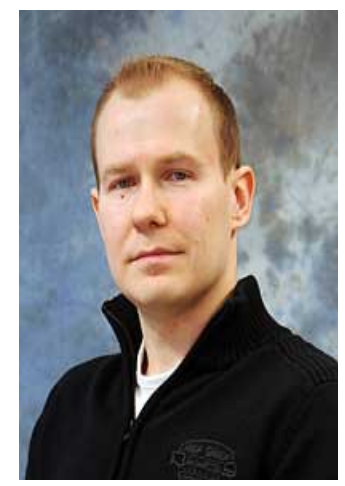

Jari Luomala is a project researcher at the University of Jyväskylä, Kokkola University Consortium Chydenius. He received the B.Eng. in software engineering from the Central Ostrobothnia Polytechnic, Finland, in 2001, and the M.Sc. in information technology from the University of Jyväskylä, Finland, in 2011. He is currently a doctoral student in mathematical information technology at the Faculty of Information Technology, University of Jyväskylä. His main research interests include localization in wireless sensor networks.

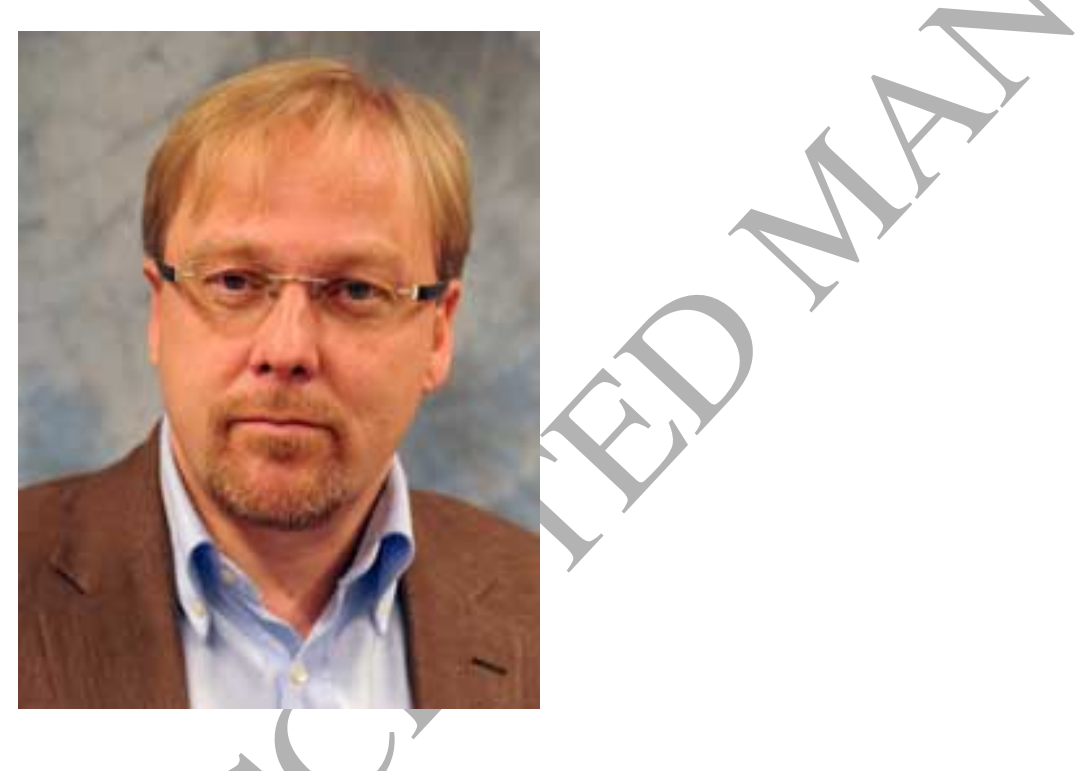

Ismo Hakala is a professor of computer science at the Kokkola University Consortium Chydenius (KUC), University of Jyväskylä, Finland. He received his M.Sc. (1989), Ph. Lic. (1992), and Ph.D. (1997) degrees in mathematics at the University of Oulu, Finland. He joined at the University of Jyväskylä in 1999 . He is currently the head of of Information Technology Unit at the KUC and leads the research group which is focusing on intelligent and autonomous sensor systems with application on environmental monitoring, healthcare and smart cities. His current research interests lie in wireless sensor networks with emphasis on data processing, localization algorithms, power saving mechanisms, and the design and performance evaluation of wireless sensor systems. 